\title{
Effective Hamiltonian and low-lying energy clustering patterns of four-sublattice antiferromagnets
}

\author{
N.-G. Zhang ${ }^{a}$, C. L. Henley ${ }^{a}$, C. Rischel ${ }^{b}$, and K. Lefmann ${ }^{c}$ \\ ${ }^{a}$ Dept. of Physics, Cornell University, Ithaca NY 14853-2501 \\ ${ }^{b}$ Niels Bohr Institute, University of Copenhagen, Blegdamsvej 17, DK-2100 Kbenhavn, Denmark \\ ${ }^{c}$ Department of Materials Research, Risø National Laboratory, DK-4000 Roskilde, Denmark
}

\begin{abstract}
We study the low-lying energy clustering patterns of quantum antiferromagnets with $p$ sublattices (in particular $p=4$ ). We treat each sublattice as a large spin, and using second-order degenerate perturbation theory, we derive the effective (biquadratic) Hamiltonian coupling the $p$ large spins. In order to compare with exact diagonalizations, the Hamiltonian is explicitly written for a finite-size lattice, and it contains information on energies of excited states as well as the ground state. The result is applied to the face-centered-cubic Type I antiferromagnet of spin $1 / 2$, including secondneighbor interactions. A 32-site system is exactly diagonalized, and the energy spectrum of the low-lying singlets follows the analytically predicted clustering pattern.
\end{abstract}

PACS numbers: 75.10.Jm, 03.65.Sq, 75.40.Mg, 75.30.Kz

\section{INTRODUCTION}

Many frustrated isotropic antiferromagnets develop long-range order, but their classical ground states have nontrivial degeneracies, so that the true (quantum) spin ordering pattern is not obvious and may be decided by small perturbations. Exact diagonalization calculations have been carried out on finite systems and the low-lying eigenenergies show interesting clustering patterns. In this paper we develop an effective Hamiltonian approach to studying energy clustering for a finite-size lattice. The approximation that we use is to divide the system into sublattices following the classical ground states, represent the spins in each sublattice by one large spin, and account for the fluctuations within sublattices by an effective Hamiltonian, which couples the large sublattice spins to each other. This effective Hamiltonian is much more manageable (as far as exact diagonalization is concerned) than the original Hamiltonian. From this effective Hamiltonian, using notions of Bohr-Sommerfeld quantization, tunneling, and the geometrical phase, one can predict the energy patterns of low-lying singlet states of a small system, as may be "observed" in exact diagonalizations. Recently, in Ref. 1. two of us studied the triangular antiferromagnet with nearest and next nearest neighbor interactions and compared analytical predictions with exact diagonalization results in Ref. 2. In this paper, we present a systematic derivation of the (biquadratic) effective Hamiltonian for a class of antiferromagnets (this was explained in Ref. 11 merely by a footnote). And we check its predictions on the eigenenergy clustering patterns by carrying out exact diagonalizations of the fcc Type I antiferromagnet. We will also correct some numerical mistakes in a table in Ref. 1, resulting in a much better agreement between diagonalization and analytical results for the triangular lattice.

We start with a general Heisenberg Hamiltonian

$$
\hat{\mathcal{H}}\left(\left\{\mathbf{s}_{i}\right\}\right)=\frac{1}{2} \sum_{i j} J_{i j} \mathbf{s}_{i} \cdot \mathbf{s}_{j}
$$

where $i$ and $j$ run over $N$ lattice sites and each $\mathbf{s}_{i}$ has quantum spin $s . \quad J_{i j}$ is the coupling constant between the sites $i$ and $j$ and is equal to $J_{m}$ when $i$ and $j$ are $m$-th nearest neighbors $(m=1,2, \ldots)$.

We assume that the ground state of $\hat{\mathcal{H}}$ has long-range antiferromagnetic order (of the spin directions). In the simplest antiferromagnets (such as the square or triangular lattice with nearest-neighbor interactions), the classical ground state is unique (modulo rotations) and the quantum ground state has the same ordering pattern. The antiferromagnets of interest here have continuous degeneracies - not due to symmetry - which are typically broken by quantum fluctuations 3 .

The present work applies to a subset of all classically degenerate Heisenberg antiferromagnets, which we call " $p$-sublattice." This means the lattice divides into $p$ equivalent sublattices, and the classical ground states are precisely those in which (i) the spins are parallel within each sublattice; (ii) the vector sum of all the sublattice magnetizations is zero. The two main examples that belong to this class and have motivated this study are the triangular and fcc Type-I systems with appropriate $J_{1}$ and $J_{2}$ interactions. The $p$-sublattice antiferromagnets are discussed in more detail below (Sec. [II).

The effective Hamiltonian $\hat{\mathcal{H}}_{\text {eff }}$ is a function of the $p$ sublattice spins. The simplest terms of the required symmetry are of biquadratic form, and any more complicated form would be difficult to use in an analytic calculation. This effective Hamiltonian is derived by expanding around mean-field theory. That is, one invents a "meanfield Hamiltonian" $\hat{\mathcal{H}}_{\mathrm{MF}}$ which has the same classical ground state as the true one, and which has an exactly known quantum ground state. The full Hamiltonian is then written as

$$
\hat{\mathcal{H}} \equiv \hat{\mathcal{H}}_{\mathrm{MF}}+\delta \hat{\mathcal{H}}
$$

and $\hat{\mathcal{H}}_{\text {eff }}$ is obtained by second-order perturbation theory in $\delta \hat{\mathcal{H}}$.

Mean-field theory may be set up in two different ways 
and these produce two different recipes for $\hat{\mathcal{H}}_{\text {eff }}$ The first approach was followed by Larson and Henleys, generalizing a formula of Long 6 . There, $\hat{\mathcal{H}}_{\mathrm{MF}}$ consists of local fields that fix each spin in its classical direction; the result is a derivation of $\hat{\mathcal{H}}_{\text {eff }}$, usable for any antiferromagnet with a nontrivial degeneracies, but only for an infinite system. In this paper, we follow the second approach to mean-field theory: that is, the zeroth-order Hamiltonian is an infinite-range model in which the individual spins and their products are replaced by the sublattice averages. This has the advantage that the finite- $N$ effects are accounted for (this is important for comparing with exact-diagonalization results).

The calculation proceeds in several stages. The first stage is to map each sublattice in the $N$-spin system approximately to just one spin of maximum length 1 The net energy from the neglected spin fluctuations of the spins in each sublattice (away from the perfectly aligned state), is approximated by an effegtive biquadratic interaction favoring collinear states $\mathbf{3}$. Such interactions appeared first in the theory of selection among degenerate classicaloround states (the so-called "order-by-disorder" effect).

Mathematically, our calculation involves two steps. First, operator equivalents that operate on sublattice spin states are obtained for one spin and the product of two spins. This step is purely mathematical and follows from the Wigner-Eckart theorem concerning the addition of angular momenta. The second step is summing the operator equivalents over the lattice, and this step depends on the geometric relationship of the antiferromagnet's sublattices.

In this paper, we derive the biquadratic effective Hamiltonian and apply it to the fcc Type I antiferromagnet (with nearest- and next-nearest-neighbor interactions). We generalize this derivation to treat arbitrary (translation-invariant) exchange couplings, in particular to properly handle couplings which connect two spins of the same sublattice (such as $J_{2}$ in the fcc Type I antiferromagnet). Combining this with the remaining results of Ref. 1 on Bohr-Sommerfeld semiclassical quantization and geometrical phase, we find that the expected splitting pattern for the fcc Type I indeed agrees with numerical diagonalization results for $N=32$ sites. (See Ref. 9 for a more detailed description of numerical diagonalization of fcc systems and Ref. 10 for an earlier study of another spin system, a three-spin cluster, using many of the key semiclassical ideas here.)

This paper is organized as follows: we first introduce the class of $p$-sublattice antiferromagnets and give some examples (Sec. [II); we then motivate and give the meanfield Hamitonian (Sec. III); next, the biquadratic effective Hamiltonian is derived from second-order perturbation theory (this is the core of the paper) (Sec. IV). We then apply semiclassical considerations to the resulting effective Hamiltonian (Sec. V) analytically calculating the energy-level pattern. The analytic result for the type-I fcc antiferromagnet, as well as a convenient four- spin exact diagonalization based on the same effective Hamiltonian, is compared with exact diagonalization of a 32-site system (Sec. VI); finally, we make some closing observations (Sec. VII).

\section{THE $P$-SUBLATTICE ANTIFERROMAGNETS}

We will set up a formalism that includes all realizations of $p$-sublattice antiferromagnets. It is convenient to define a geometric factor $z_{\alpha \beta}^{m}$, as the number of $m$-th neighbors on sublattice $\alpha$ of a site on sublattice $\beta$. (We will consistently use Greek indices $\alpha, \beta$ to label sublattices and $\mu, \nu, \rho$ to label spin components.) Notice that $z_{\alpha \beta}^{m}$ is not an attribute of the Hamiltonian (1.1), but of the geometrical arrangement of sublattices within the system.

Unless specifically noted, we will assume (and need) sublattice-pair permutation symmetry. This means that if we perform any of the $p$ ! permutations of the sublattice labels, the geometric relationship between any pair $(\alpha, \beta)$ will be unchanged (modulo lattice symmetries such are translations, rotations, and reflections).

The classical ground state manifold is labeled by the unit vectors $\left\{\hat{\mathbf{m}}_{\alpha}\right\}, \alpha=1, \ldots, p$ for the directions of the sublattices. Since we seek a quantum-mechanical Hamiltonian, however, it should be expressed in terms of the corresponding operators, which (apart from length normalization) are the sublattice spins,

$$
\mathbf{S}_{\alpha}=\sum_{i: \alpha(i)=\alpha} \mathbf{s}_{i},
$$

where $\alpha(i)=1,2, \ldots, p$ gives the sublattice index of the spin $\mathbf{s}_{i}$. Here the sum is over $\tilde{N} \equiv N / p$ sites within the sublattice $\alpha$. Each $\mathbf{S}_{\alpha}$ can be in states with total spin ranging from zero to $\tilde{S} \equiv \tilde{N} s$. The classical state corresponds to a coherent state in which every $\mathbf{s}_{i}$ in sublattice $\alpha$ is aligned with $\hat{\mathbf{m}}_{\alpha}$, hence each sublattice spin has its maximum length $\tilde{S}$.

Our final goal will be an effective Hamiltonian $\hat{\mathcal{H}}_{\text {eff }}$ defined on the sub-Hilbert-space of " $p$-sublattice states", being all states in which every sublattice spin has maximum length, $\mathbf{S}_{\alpha}^{2}=\tilde{S}(\tilde{S}+1)$; thus $\hat{\mathcal{H}}_{\text {eff }}$ will have the form of a spin Hamiltonian. (Note that these " $p$-sublattice states" have maximum length. The sublattice magnetization is not determined and will be decided by diagonalizating the secular Hamiltonian matrix as developed in the degenerate perturbation below.) Along the way, we will often deal with the Hilbert space of one particular sublattice - e.g. the operator equivalents are computed in this space. In particular, we let $\left|\phi_{\alpha}\right\rangle$ mean any eigenstate with $\mathbf{S}_{\alpha}^{2}=\tilde{S}(\tilde{S}+1)$ (maximum length) and simultaneously a definite $S_{\alpha}^{z}$. The direct products of such states make up the basis for $p$-sublattice states. On the other hand, $\left|\psi_{\alpha}\right\rangle$ will be used for a simultaneous eigenstate of $\mathbf{S}_{\alpha}^{2}$ and $S_{\alpha}^{z}$ with any total spin, $0 \leq S_{\alpha} \leq \tilde{S}$.

The chief concrete examples hare $A=4$, being: (i) the $J_{1}-J_{2}$ triangular antiferromagnet, 2.11] with $J_{1}>0$ and 
second-neighbor coupling $J_{2} \in\left(J_{1} / 8, J_{1}\right)$ - the effective Hamiltonian for this case was anpunced in Ref. 1; (ii) the Type I fcc antiferromagnet 1213 with $J_{1}>0, J_{2}<0$. In either example, there are only nearest and nextnearest neighbor interactions $\left(J_{m}=0\right.$ for $\left.m>2\right)$; the Type I fcc differs from the triangular case in that the next-nearest-neighbor couplings $\left(z^{2}\right)$ connects sites of the same sublattice. (Table I, below, includes the $z_{\alpha \beta}^{m}$ values for these lattices.) The honeycomb and pyrochlore lattices also support four-sublattice states for appropriately chosen exchange couplings $\left(J_{1}\right.$ and $\left.J_{2}\right)$. After allowing for rotations, a 4-sublattice antiferromagnet still has a two-parameter family of classical ground states. $11-13$

The case $p=3$ is less interesting: there is just one way to add up three equal-length spins to make total spin zero, so the classical or "mean-field" ground state is already unique. But our approach would be applicable to the three-sublattice antiferromagnet in an external field, in which case the ground state does have nontrivial degeneracy.

There exist $p$-sublattice antiferromagnets with $p>4$, and possessing "sublattice-pair permutation symmetry", although their Hamiltonians are physically improbable. For example, the $p=5$ case is realized by the $\sqrt{5} \times \sqrt{5}$ sublattice; in a square lattice without reflection symmetry. The ground state has five sublattices, e.g., when the couplings to the $\pm[2,1]$ and $\pm[1,-2]$ fourth neighbors are strongly ferromagnetic, and the nearest neighbor couplings are antiferromagnetic. A similar construction with $p=7$ may be made using the $\sqrt{7} \times \sqrt{7}$ sublattice of a triangular lattice.

\section{MEAN-FIELD HAMILTONIAN}

The objective of this section is to construct from $\hat{\mathcal{H}}$ of Eq. (1.1) a "mean-field" Hamiltonian $\hat{\mathcal{H}}_{\mathrm{MF}}$ that is defined on the entire Hilbert space but expressible in terms of the sublattice spins $\left\{\mathbf{S}_{\alpha}\right\}$. In other words, it is a projection of the exact Hamiltonian onto a kind of infinite-range Hamiltonian. We will show that the ground states of $\hat{\mathcal{H}}_{\mathrm{MF}}$ are all the $p$-sublattice states; this high degeneracy corresponds to the classical degeneracy. The real value of $\hat{\mathcal{H}}_{\mathrm{MF}}$ is that we can split the Hamiltonian in form (1.2) and obtain $\hat{\mathcal{H}}_{\text {eff }}$ via second-order perturbation in $\delta \hat{\mathcal{H}}$ (see Sec. [V].

The eigenstates of the lattice system that correspond one-to-one to those of our $p$-spin effective Hamiltonian are not literally superpositions of the " $p$-sublattice states." The true eigenstates include quantum fluctuations that admix other states (as is implicit in our second-order perturbation), so the overlap with the $p$ sublattice subspace must decrease exponentially with increasing system size $N$; indeed the authors of Ref. 2 computed the overlap and found it small 14 The low-energy states of the lattice antiferromagnet are related to the $p$-spin system's eigenstates much as the excited states of a Fermi liquid are related to those of noninteracting electrons. 15 In this paper, our main interest is in analyzing the clustering patterns of the low-lying energy spectrum. We will focus on calculating eigenenergies, not magnetization expectations.

The mean-field Hamiltonian $\hat{\mathcal{H}}_{\mathrm{MF}}$, to be a function only of the sublattice totals $\left\{\mathbf{S}_{\alpha}\right\}$, must be symmetric under any way of permuting the spins within a sublattice. Furthermore, the states that are symmetric under such permutations are just the $p$-sublattice states, and $\hat{\mathcal{H}}_{\text {MF }}$ ought to have the same expectation as $\hat{\mathcal{H}}$ in such a state. (This will be demonstrated in the next section.)

The unique way to project $\hat{\mathcal{H}}$ onto $\hat{\mathcal{H}}_{\mathrm{MF}}$ and satisfy these conditions is to average each term $\mathbf{s}_{i} \cdot \mathbf{s}_{j}$ over permutations of the sublattice(s) to which the sites $i$ and $j$ belong. There are two cases. First, the two spins are on different sublattices $(\alpha(i) \neq \alpha(j))$; then we simply replace $\mathbf{s}_{i} \cdot \mathbf{S}_{j} \rightarrow\left(\mathbf{S}_{\alpha(i)} / \tilde{N}\right) \cdot\left(\mathbf{S}_{\alpha(j)} / \tilde{N}\right)$. Second, the spins are on the same sublattice $(\alpha(i)=\alpha(j)=\alpha$ with $i \neq j)$; in this case there are $\tilde{N}(\tilde{N}-1)$ pairs of such scalar products within one sublattice. (No permutation can ever make $i=j$.) We use the identity $\mathbf{S}_{\alpha}^{2}=\tilde{N} s(s+1)+\sum_{i \neq j} \mathbf{s}_{i} \cdot \mathbf{s}_{j}$ to obtain that $\mathbf{s}_{i} \cdot \mathbf{s}_{j} \rightarrow\left(\mathbf{S}_{\alpha}^{2}-\tilde{N} s(s+1)\right) / \tilde{N}(\tilde{N}-1)$ in this case. (When $S_{\alpha}=\tilde{S}$, i.e., maximum length, this average is simply $s^{2}$; restricted to the maximum-spin subspace, the projection we are doing here agrees with the operator equivalent of $\mathbf{s}_{i} \cdot \mathbf{s}_{j}$, defined in (4.6), below.) Finally, putting these two kinds of terms together and performing the sum, we get

$$
\begin{aligned}
\hat{\mathcal{H}}_{\mathrm{MF}} & =\frac{1}{2} \sum_{\alpha \neq \beta} \tilde{J}_{\alpha \beta} \mathbf{S}_{\alpha} \cdot \mathbf{S}_{\beta} \\
& +\frac{1}{2} \sum_{\alpha} \tilde{J}_{\alpha \alpha} \frac{\tilde{N}}{\tilde{N}-1}\left(\mathbf{S}_{\alpha}^{2}-\tilde{N} s(s+1)\right),
\end{aligned}
$$

where

$$
\tilde{J}_{\alpha \beta} \equiv \sum_{m}\left(J_{m} z_{\alpha \beta}^{m}\right) / \tilde{N}
$$

We restrict our attention to the class of $p$-sublattice antiferromagnets with sublattice-pair permutation symmetry, so $z_{\alpha \beta}^{m}=z_{12}^{m}$ for each $m$ and $\alpha \neq \beta$ and $z_{\alpha \alpha}^{m}=z_{11}^{m}$. Thus $\tilde{J}_{\alpha \beta}=\tilde{J}_{12}$ for all $\alpha \neq \beta$ and $\tilde{J}_{\alpha \alpha}=\tilde{J}_{11}$ for all $\alpha$ 's. The mean-field Hamiltonian becomes,

$$
\begin{aligned}
\hat{\mathcal{H}}_{\mathrm{MF}}= & \frac{1}{2} \tilde{J}_{12}\left(\mathbf{S}_{\mathrm{tot}}^{2}-\sum_{\alpha} \mathbf{S}_{\alpha}^{2}\right) \\
& +\frac{1}{2} \tilde{J}_{11} \frac{\tilde{N}}{\tilde{N}-1}\left(\sum_{\alpha} \mathbf{S}_{\alpha}^{2}-p \tilde{N} s(s+1)\right) \\
= & \frac{1}{2} \tilde{J}_{12} \mathbf{S}_{\mathrm{tot}}^{2}-\frac{1}{2} \tilde{J}_{S} \sum_{\alpha} \mathbf{S}_{\alpha}^{2}-\frac{1}{2} p \tilde{J}_{11} \frac{\tilde{N}^{2}}{\tilde{N}-1} s(s+1),
\end{aligned}
$$

where $\mathbf{S}_{\text {tot }}=\sum_{\alpha} \mathbf{S}_{\alpha}$ and we have also defined 


$$
\tilde{J}_{S}=\tilde{J}_{12}-\frac{\tilde{N}}{\tilde{N}-1} \tilde{J}_{11}
$$

Next we consider the ground states for $\hat{\mathcal{H}}_{\mathrm{MF}}$, which we denote $|\Phi\rangle$. For this, we further restrict to the situation where $\tilde{J}_{S}>0$, i.e., the coefficient in front of $\sum \mathbf{S}_{\alpha}^{2}$ in (3.4) is positive. (This condition is satisfied by both the triangular antiferromagnet $\left(\tilde{J}_{11}=0\right)$ and the fcc type-I case $\left(\tilde{J}_{12}>0\right.$ and $\left.\tilde{J}_{11}<0\right)$. See Table 1 for a collection of relevant parameters.)

Then $|\Phi\rangle$ should obviously satisfy $\mathbf{S}_{\text {tot }}^{2}|\Phi\rangle=0$ and $\mathbf{S}_{\alpha}^{2}|\Phi\rangle=\tilde{S}(\tilde{S}+1)|\Phi\rangle$ for every $\alpha$. In other words, the ground states of $\hat{\mathcal{H}}_{\mathrm{MF}}$ are just the $p$-sublattice states which are also total singlets, so we call them " $p$-sublattice singlet states." (A similar result is obtained for the classical ground state: that each of the $p$ sublattice spin vectors is at maximum length and their vector sum is zero.) For $p=4$, there are $2 \tilde{S}+1$ such singlets: 2 for $p=5$ and $\tilde{S}$ is an integer, there are $5 \tilde{S}(\tilde{S}+1) / 2+1$ (see Appendix A). Lecheminant et al. 2 already recognized that, in the $J_{1}$ $J_{2}$ triangular antiferromagnet, the lowest energy singlets correspond to the four-sublattice singlet states.

We emphasize here that we have a degenerate family of singlets. These states have the rotational symmetry as the true finite- $\mathrm{N}$ ground state is known to have (i.e., they are singlets). The sublattice spins have maximum length but the $\mathrm{z}$ components (magnetization expectations) are not determined. Which linear combination of these states best represents the ground state is only settled by the degenerate perturbation calculation that follows, which diagonalizes an effective Hamiltonian within this degenerate family of singlet states.

The ground state energy of (3.4) is

$$
E_{\mathrm{MF}}=\frac{1}{2} p\left[\tilde{J}_{11} \tilde{S}^{2}-\tilde{J}_{12} \tilde{S}(\tilde{S}+1)\right] .
$$

Table If gives $E_{\mathrm{MF}}$ for the triangular and fcc lattices.

It is necessary to correct this mean-field Hamiltonian by $\hat{\mathcal{H}}_{\text {eff }}$, the effective biquadratic Hamiltonian, 0 to predict the sequence of quantum numbers of the low-lying states, and the clustering pattern of their eigenenergies.

\section{BIQUADRATIC EFFECTIVE HAMILTONIAN}

The calculation of the second-order effective Hamiltonian $\hat{\mathcal{H}}_{\text {eff }}\left(\left\{\mathbf{S}_{\alpha}\right\}\right)$ will be presented now, and it is the central analytic result of this paper. We will use the second-order degenerate perturbation theory 16 for $\delta \hat{\mathcal{H}} \equiv$ $\hat{\mathcal{H}}-\hat{\mathcal{H}}_{\mathrm{MF}}$,

$$
\left\langle\Phi^{\prime}\left|\hat{\mathcal{H}}_{\mathrm{eff}}\right| \Phi\right\rangle=-\sum_{\Psi} \frac{\left\langle\Phi^{\prime}|\delta \hat{\mathcal{H}}| \Psi\right\rangle\langle\Psi|\delta \hat{\mathcal{H}}| \Phi\rangle}{E_{\Psi}-E_{\mathrm{MF}}},
$$

where $|\Phi\rangle$ and $\left|\Phi^{\prime}\right\rangle$ are both $p$-sublattice singlet states (i.e., in the ground state manifold of $\hat{\mathcal{H}}_{\mathrm{MF}}$ ) and $|\Psi\rangle$ is an excited state of $\hat{\mathcal{H}}_{\mathrm{MF}}$. In the process, we will also show that this choice of mean field Hamiltonian enables the first-order correction to vanish, i.e.,

$$
\left\langle\Phi^{\prime}|\delta \hat{\mathcal{H}}| \Phi\right\rangle=0 .
$$

It is easy to show that

$$
\delta \hat{\mathcal{H}}=\delta \hat{\mathcal{H}}_{\text {diff }}+\delta \hat{\mathcal{H}}_{\text {same }},
$$

where,

$$
\begin{aligned}
& \delta \hat{\mathcal{H}}_{\mathrm{diff}}=\frac{1}{2} \sum_{m} J_{m} \sum_{\substack{m-\operatorname{th}(i j) \\
\alpha(i) \neq \alpha(j)}} \delta \mathbf{s}_{i} \cdot \delta \mathbf{s}_{j} \\
& =\frac{1}{2} \sum_{m} J_{m}\left(\sum_{\substack{m-\operatorname{th}(i j) \\
\alpha(i) \neq \alpha(j)}} \mathbf{s}_{i} \cdot \mathbf{s}_{j}-\frac{1}{\tilde{N}} \sum_{\alpha \neq \beta} z_{\alpha \beta}^{m} \mathbf{S}_{\alpha} \cdot \mathbf{S}_{\beta}\right),
\end{aligned}
$$

and,

$$
\delta \hat{\mathcal{H}}_{\text {same }}=\frac{1}{2} \sum_{m} J_{m} \sum_{\substack{m-\operatorname{th}(i j) \\ \alpha(i)=\alpha(j) \\=\alpha}}\left(\mathbf{s}_{i} \cdot \mathbf{s}_{j}-\frac{\mathbf{S}_{\alpha}^{2}-\tilde{N} s(s+1)}{\tilde{N}(\tilde{N}-1)}\right),
$$

and $\delta \mathbf{s}_{i}=\mathbf{s}_{i}-\mathbf{S}_{\alpha(i)} / \tilde{N}$. Here the subscript "diff" means that the sum is over $(i j)$ pairs on different sublattices and "same" means that they are on the same sublattices; also, " $m-\operatorname{th}(i j)$ " means a sum is restricted to pairs $(i, j)$ which are $m$-th neighbors. We should consider what kind of excited states $|\Psi\rangle$ are present in the sum of the secondorder perturbation theory (4.1). Because this consideration in part relies on the Wigner-Eckart theorem and the concept operator equivalent, we will turn to these mathematical concepts now.

\section{A. Operator equivalents}

The general problem of this sort of calculation is to take an operator defined in a large Hilbert space, restrict this Hilbert space to a smaller one, and re-express its action by an operator acting on the restricted space. The latter operator will be called the "operator equivalent" of the first one. In the present paper, we start from an operator $\hat{O}\left(\left\{\mathbf{s}_{i}\right\}\right)$ that acts on all states of the spins $\left\{\mathbf{s}_{i}\right\}$ belonging to sublattice $\alpha$ and convert it into an operatpr $\hat{o}\left(\mathbf{S}_{\alpha}\right)$ acting on states of a single net sublattice spin $\mathbf{S}_{\alpha} \cdot 17$

The obvious tool for this calculation is the WignerEckart theorem 18 which deals with angular momenta addition. It says that among states of a single spin multiplet (such as the maximum-spin states $\left|\phi_{\alpha}\right\rangle$ ), any operator that transforms as a particular representation of the rotation group, has matrix elements given by Clebsch-Gordan coefficients, apart from a single constant factor setting the overall scale. Hence we can replace that operator by 
a simpler one belonging to the same representation: here, a polynomial $\hat{o}\left(\mathbf{S}_{\alpha}\right)$. There is a unique such polynomial for each of the rotational symmetries (scalar, vector, or traceless-symmetric tensor). Specifically, we will be given operators $\hat{O}\left(\left\{\mathbf{s}_{i}\right\}\right)$, with a given symmetry, and write their matrix elements $\left\langle\phi_{\alpha}^{\prime}\left|\hat{O}\left(\left\{\mathbf{s}_{i}\right\}\right)\right| \phi_{\alpha}\right\rangle=c\left\langle\phi_{\alpha}^{\prime}\left|\hat{o}\left(\mathbf{S}_{\alpha}\right)\right| \phi_{\alpha}\right\rangle$ where $c$ is a constant that depends on $S_{\alpha}$, and possibly on $i$, but not on the $z$-component of the spin (see e.g., Ref. 19). In our calculation, we restrict our attention to the maximum spin manifold $S_{\alpha}=\tilde{S}$ because it turns out that we will need only matrix elements between the quantum $p$-sublattice singlet states $|\Phi\rangle$ and $\left|\Phi^{\prime}\right\rangle$, where $S_{\alpha}=\tilde{S}$ for all $\alpha$; in any case, when $\mathbf{S}_{\alpha}<\tilde{S}$ there is more than one multiplet and the Wigner-Eckart theorem is not enough to specify the matrix elements.

The results for scalar and vector operators are,

$$
\begin{gathered}
\left\langle\phi_{\alpha}^{\prime}\left|\mathbf{s}_{i} \cdot \mathbf{s}_{j}\right| \phi_{\alpha}\right\rangle=s^{2}\left\langle\phi_{\alpha}^{\prime} \mid \phi_{\alpha}\right\rangle, \\
\quad \alpha(i)=\alpha(j)=\alpha, \quad i \neq j, \quad S_{\alpha}=\tilde{S} \\
\left\langle\phi_{\alpha}^{\prime}\left|s_{i}^{\mu}\right| \phi_{\alpha}\right\rangle=\frac{1}{\tilde{N}}\left\langle\phi_{\alpha}^{\prime}\left|S_{\alpha}^{\mu}\right| \phi_{\alpha}\right\rangle, \\
\alpha(i)=\alpha, \quad S_{\alpha}=\tilde{S} .
\end{gathered}
$$

The constant coefficients, $c=s^{2}$ for the scalar operator equivalent and $c=1 / \tilde{N}$ for the vector operator equivalent, were found by explicitly calculating the matrix elements on both sides for a particular choice of states: $\left|\phi_{\alpha}\right\rangle$ and $\left|\phi_{\alpha}^{\prime}\right\rangle$ are both the state with all spins aligned along $+z$, i.e., $S_{\alpha}^{z}=\tilde{S}$. This is the most convenient choice to simplify the algebra.

As noted above, every maximum-spin state $\left|\phi_{\alpha}\right\rangle$ is symmetric under permutations of the sites of sublattice $\alpha$, hence our operator equivalent expressions (4.6) and (4.7) cannot depend on $i$ and $j$. This symmetry could be used in place of the Wigner-Eckart theorem to derive eqs. (4.6) and (4.7), but only the Wigner-Eckart approach works in the tensor operator case (Sec. IV D, below).

\section{B. Energy denominators}

Now we are ready to compute the matrix elements $\langle\Psi|\delta \hat{\mathcal{H}}| \Phi\rangle$ and denominators $E_{\Psi}-E_{\mathrm{MF}}$ for the excited states in the perturbation sum (4.1). First we address $\delta \hat{\mathcal{H}}_{\text {same }}$ which can be considered as acting on states of sublattice $\alpha,\left|\phi_{\alpha}\right\rangle$ or $\left|\psi_{\alpha}\right\rangle$. Notice that the operator $\mathbf{s}_{i} \cdot \mathbf{s}_{j}$ with $\alpha(i)=\alpha(j)=\alpha$ commutes with $\mathbf{S}_{\alpha}^{2}$. From (4.5), therefore, $\left[\delta \hat{\mathcal{H}}_{\text {same }}, \mathbf{S}_{\alpha}^{2}\right]=0$ for all $\alpha$. This means that, if the states $\left|\phi_{\alpha}\right\rangle$ and $\left|\psi_{\alpha}\right\rangle$ have different spin, the matrix element $\left\langle\psi_{\alpha}\left|\delta \hat{\mathcal{H}}_{\text {same }}\right| \phi_{\alpha}\right\rangle$ is zero. Since in our perturbation sum $|\Phi\rangle$ is a maximum spin state, the state $\left|\phi_{\alpha}\right\rangle$ has spin $\tilde{S}$ and so $S_{\alpha}=\tilde{S}$ for $\left|\psi_{\alpha}\right\rangle$ also. Finally we have, for $\alpha(i)=\alpha(j)=\alpha$,

$$
\left\langle\psi_{\alpha}\left|\mathbf{s}_{i} \cdot \mathbf{s}_{j}-\frac{\mathbf{S}_{\alpha}^{2}-\tilde{N} s(s+1)}{\tilde{N}(\tilde{N}-1)}\right| \phi_{\alpha}\right\rangle
$$

$$
=\left\langle\psi_{\alpha}\left|\mathbf{s}_{i} \cdot \mathbf{s}_{j}-s^{2}\right| \phi_{\alpha}\right\rangle=0,
$$

where the last step relies on the scalar operator equiva-

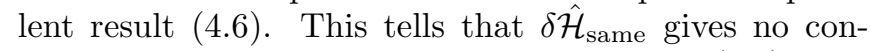
tribution to the second-order perturbation (4.1), i.e., $\left\langle\Psi\left|\delta \hat{\mathcal{H}}_{\text {same }}\right| \Phi\right\rangle=0$. Of course, the argument here also shows that $\delta \hat{\mathcal{H}}_{\text {same }}$ gives no contribution to the first-order perturbation (4.2), i.e., $\left\langle\Phi^{\prime}\left|\delta \hat{\mathcal{H}}_{\text {same }}\right| \Phi\right\rangle=0$.

In the same way, we turn to the contribution by $\delta \hat{\mathcal{H}}_{\text {diff }}$. It is easy to show that $\left[\left(\delta \mathbf{s}_{i} \cdot \delta \mathbf{s}_{j}\right), \mathbf{S}_{\text {tot }}^{2}\right]=0$ which gives $\left[\delta \hat{\mathcal{H}}_{\text {diff }}, \mathbf{S}_{\text {tot }}^{2}\right]=0$. Since $|\Phi\rangle$ has $\mathbf{S}_{\text {tot }}^{2}=0$, it follows that $\mathbf{S}_{\text {tot }}^{2}=0$ for $|\Psi\rangle$ also, otherwise $\left\langle\Psi\left|\delta \hat{\mathcal{H}}_{\text {diff }}\right| \Phi\right\rangle$ would be zero.

Furthermore, that matrix element can be broken into terms of form $\left\langle\psi_{1} \psi_{2} \psi_{3} \psi_{4}\left|\delta \mathbf{s}_{i} \cdot \delta \mathbf{s}_{j}\right| \phi_{1} \phi_{2} \phi_{3} \phi_{4}\right\rangle$. Assume without loss of generality that $\alpha(i)=1, \alpha(j)=$ 2 , so the matrix element factors as $\left\langle\psi_{1}\left|\delta \mathbf{s}_{i}\right| \phi_{1}\right\rangle$. $\left\langle\psi_{2}\left|\delta \mathbf{s}_{j}\right| \phi_{2}\right\rangle\left\langle\psi_{3} \mid \phi_{3}\right\rangle\left\langle\psi_{4} \mid \phi_{4}\right\rangle$. Consider the first factor: as ) defined above, $\left|\phi_{1}\right\rangle$ has $S_{1}=\tilde{S}$, and the raising and lowering operators in $\delta \mathbf{s}_{i}$ can change $S_{\alpha}$ by $+1,0$, or -1 only. An increase $\tilde{S} \rightarrow \tilde{S}+1$ is obviously impossible since $\tilde{S}$ is the maximum spin. Also, when $\left|\psi_{1}\right\rangle$ has the same length spin, $\tilde{S}$, the matrix element is zero by the construction $\delta \mathbf{s}_{i}=\mathbf{s}_{i}-\mathbf{S}_{\alpha(i)} / \tilde{N}$, as found using the operator equivalent (4.7), Hence, by elimination, the only states $\left|\psi_{1}\right\rangle$ that can give any nonzero matrix element in $\left\langle\psi_{1}\left|\delta \mathbf{s}_{i}\right| \phi_{1}\right\rangle$ must have spin $\tilde{S}-1$; the same holds for $\left|\psi_{2}\right\rangle$, whereas $\left|\psi_{3}\right\rangle$ and $\left|\psi_{4}\right\rangle$ obviously have the same spin $\tilde{S}$ as do $\left|\phi_{3}\right\rangle$ and $\left|\phi_{3}\right\rangle$.

Finally, every term of $\delta \hat{\mathcal{H}}_{\text {diff }}$ contains products of two such operators $\delta \mathbf{s}_{i} \cdot \delta \mathbf{s}_{j}$, so $\left\langle\Psi\left|\delta \hat{\mathcal{H}}_{\text {diff }}\right| \Phi\right\rangle$ is nonzero only if $|\Psi\rangle$ has exactly two of the $p$ spins reduced by one. This gives

$$
\begin{aligned}
E_{\Psi}=- & \frac{1}{2} \tilde{J}_{S}((p-2) \tilde{S}(\tilde{S}+1)+2(\tilde{S}-1) \tilde{S}) \\
& -\frac{1}{2} p \tilde{J}_{11} \frac{\tilde{N}^{2}}{\tilde{N}-1} s(s+1) .
\end{aligned}
$$

Subtracting the ground state energy $E_{\mathrm{MF}}$ (3.6), the energy denominator in the perturbation sum (4.1) that can give nonzero contribution is always

$$
E_{\Psi}-E_{\mathrm{MF}}=2 \tilde{J}_{S} \tilde{S}
$$

This argument also gives that $\left\langle\Phi^{\prime}\left|\delta \hat{\mathcal{H}}_{\text {diff }}\right| \Phi\right\rangle=0$ within the ground states of $\hat{\mathcal{H}}_{\mathrm{MF}}$. Together with the result $\left\langle\Phi^{\prime}\left|\delta \hat{\mathcal{H}}_{\text {same }}\right| \Phi\right\rangle=0$ obtained earlier in this section, we conclude that the first-order correction to $\hat{\mathcal{H}}_{\mathrm{MF}}$ by $\delta \hat{\mathcal{H}}$ is zero, i.e., eq. (4.2) is established.

\section{Reduction of Perturbation Sum}

We have now concluded that all the states $|\Psi\rangle$ that contribute nonzero numerator to the perturbation sum (4.1) have the same denominator. This key fact enables 
us to factor the denominators out of sum (4.1). Then we can extend the sum to run over all states $|\Psi\rangle$, not just the excited ones. (Every added term is zero, since $|\Psi\rangle$ is a maximum-spin state in the added terms, and we just showed that $\langle\Psi|\delta \hat{\mathcal{H}}| \Phi\rangle$ is always zero in that case.) Equation (4.1) then becomes

$$
\left\langle\Phi^{\prime}\left|\hat{\mathcal{H}}_{\text {eff }}\right| \Phi\right\rangle=-\frac{\left\langle\Phi^{\prime}\left|\left(\delta \hat{\mathcal{H}}_{\text {diff }}\right)^{2}\right| \Phi\right\rangle}{2 \tilde{J}_{S} \tilde{S}} .
$$

So instead of trying to sum over excited states in eq. (4.1), our goal now will be to find the operator equivalent of $\left(\delta \hat{\mathcal{H}}_{\text {diff }}\right)^{2}$ as in eq. (4.11). Squaring (4.4) gives

$$
\left\langle\Phi^{\prime}\left|\left(\delta \hat{\mathcal{H}}_{\text {diff }}\right)^{2}\right| \Phi\right\rangle=\left\langle\Phi^{\prime}\left|\frac{1}{4} \sum_{m n} J_{m} J_{n} \hat{\mathcal{O}}_{m n}\right| \Phi\right\rangle,
$$

where,

$$
\begin{aligned}
& \hat{\mathcal{O}}_{m n}=\sum_{\substack{m-\operatorname{th}(i j) \\
\alpha(i) \neq \alpha(j)}} \sum_{\substack{n-\operatorname{th}(k l) \\
\alpha(k) \neq \alpha(l)}}\left[\left(\mathbf{s}_{i} \cdot \mathbf{s}_{j}\right)\left(\mathbf{s}_{k} \cdot \mathbf{s}_{l}\right)\right. \\
& \left.-\frac{1}{\tilde{N}^{2}}\left(\sum_{\alpha \neq \beta} \mathbf{S}_{\alpha} \cdot \mathbf{S}_{\beta}\right)\left(\sum_{\gamma \neq \delta} \mathbf{S}_{\gamma} \cdot \mathbf{S}_{\delta}\right)\right]
\end{aligned}
$$

In deriving this, we have used another operator equivalent relation

$$
\left\langle\phi_{\alpha}^{\prime}\left|s_{i}^{\mu} S_{\alpha}^{\nu}\right| \phi_{\alpha}\right\rangle=\frac{1}{\tilde{N}}\left\langle\phi_{\alpha}^{\prime}\left|S_{\alpha}^{\mu} S_{\alpha}^{\nu}\right| \phi_{\alpha}\right\rangle, \quad \alpha(i)=\alpha,
$$

which is true because $\left|\phi_{\alpha}\right\rangle$, being a state of maximum $S_{\alpha}$, has the symmetry of permutation among all sites $i$ in sublattice $\alpha$.

\section{Operator equivalent of quadratic operator}

To obtain $\hat{\mathcal{H}}_{\mathrm{MF}}$, we have considered, in section III, the operator equivalents of scalar and vector operators. Here for $\hat{\mathcal{H}}_{\text {eff }}$, we need the operator equivalent of $\left(\mathbf{s}_{i} \cdot \mathbf{s}_{j}\right)\left(\mathbf{s}_{k} \cdot \mathbf{s}_{l}\right)$ which can have two of the spin operators (e.g. $\mathbf{s}_{i}$ and $\mathbf{s}_{k}$ ) on the same sublattice. For this case we will need the operator equivalents for tensor operators.

As before, to find the operator equivalent we consider a state of just one sublattice $\left|\phi_{\alpha}\right\rangle$ with the maximum spin $\tilde{S}$. Recall the decomposition of a direct product of two vector operators:

$$
s_{i}^{\mu} s_{k}^{\nu}=\frac{1}{3} \hat{A} \delta_{\mu \nu}+\epsilon_{\mu \nu \rho} \hat{V}_{\rho}+\hat{T}_{\mu \nu}
$$

where

$$
\begin{aligned}
\hat{A} & =\mathbf{s}_{i} \cdot \mathbf{s}_{k}, \quad \hat{V}_{\rho}=\frac{1}{2} \epsilon_{\mu \nu \rho} s_{i}^{\mu} s_{k}^{\nu}, \\
\hat{T}_{\mu \nu} & =\frac{1}{2}\left(s_{i}^{\mu} s_{k}^{\nu}+s_{i}^{\nu} s_{k}^{\mu}\right)-\frac{1}{3} \delta_{\mu \nu} \hat{A} .
\end{aligned}
$$

$\hat{A}$ is a scalar operator, $\hat{V}_{\rho}$ a vector operator, and $\hat{T}_{\mu \nu}$ a second-rank symmetric traceless tensor operator.

By the Wigner-Eckart theorem as stated in Sec. IV A, the operator equivalents of the three operators in (4.16) are known functions of $\mathbf{S}_{\alpha}$, apart from coefficients depending only on $(i, k)$. As before, we obtain the coefficients using the most convenient choice of states $S_{\alpha}^{z}=\tilde{S}$ (all spins aligned up) to simplify the algebra. With $\alpha(i)=\alpha(k)=\alpha$, for the scalar operator $\hat{A}$, we obtain

$$
\left\langle\phi_{\alpha}^{\prime}|\hat{A}| \phi_{\alpha}\right\rangle=\left(s^{2}+\delta_{i k} s\right)\left\langle\phi_{\alpha}^{\prime} \mid \phi_{\alpha}\right\rangle
$$

Note we can have $i=k$ here, because $i$ and $k$ come from two separate scalar products $\left(\mathbf{s}_{i} \cdot \mathbf{s}_{j}\right)\left(\mathbf{s}_{k} \cdot \mathbf{s}_{l}\right)$, whereas earlier in (4.6) we necessarily had $i \neq j$. Here $\delta_{i k}$ appears because when $i=k$ we have $\mathbf{s}_{i}^{2}=s(s+1)$. Following the same procedure for the vector and tensor operators (using all-spins-aligned states to compute coefficients), we get,

$$
\begin{aligned}
& \left\langle\phi_{\alpha}^{\prime}\left|\hat{V}_{\rho}\right| \phi_{\alpha}\right\rangle=\delta_{i k} \frac{i}{2 \tilde{N}}\left\langle\phi_{\alpha}^{\prime}\left|S_{\alpha}^{\rho}\right| \phi_{\alpha}\right\rangle, \\
& \left\langle\phi_{\alpha}^{\prime}\left|\hat{T}_{\mu \nu}\right| \phi_{\alpha}\right\rangle=\frac{1}{\tilde{N}} \frac{2 s-\delta_{i j}}{2 \tilde{S}-1} \\
& \left\langle\phi_{\alpha}^{\prime}\left|\left[\frac{1}{2}\left(S_{\alpha}^{\mu} S_{\alpha}^{\nu}+S_{\alpha}^{\nu} S_{\alpha}^{\mu}\right)-\frac{1}{3} \delta_{\mu \nu} \tilde{S}(\tilde{S}+1)\right]\right| \phi_{\alpha}\right\rangle .
\end{aligned}
$$

And putting the three terms together as in (4.15), we get,

$$
\begin{gathered}
\left\langle\phi_{\alpha}^{\prime}\left|s_{i}^{\mu} s_{k}^{\nu}\right| \phi_{\alpha}\right\rangle=\left\langle\phi_{\alpha}^{\prime}\right|\left\{\frac{1}{3}\left(s^{2}+\delta_{i k} s\right) \delta_{\mu \nu}+\delta_{i k} \frac{i}{2 \tilde{N}} \epsilon_{\mu \nu \rho} S_{\alpha}^{\rho}\right. \\
\left.+\frac{1}{\tilde{N}} \frac{2 s-\delta_{i k}}{2 \tilde{S}-1}\left[\frac{1}{2}\left(S_{\alpha}^{\mu} S_{\alpha}^{\nu}+S_{\alpha}^{\nu} S_{\alpha}^{\mu}\right)-\frac{1}{3} \delta_{\mu \nu} \tilde{S}(\tilde{S}+1)\right]\right\}\left|\phi_{\alpha}\right\rangle \\
\alpha(i)=\alpha(k)=\alpha .
\end{gathered}
$$

It may be checked that summing both sides of 4.20 ) indeed gives (4.14). As another check, notice that when $i$ and $k$ are distinct sites belonging to the same sublattice $\alpha$, the spin operators $s_{i}^{\mu}$ and $s_{k}^{\nu}$ commute. Hence the operator equivalent of $s_{i}^{\mu} s_{k}^{\nu}$ must be symmetric under exchange of $i \leftrightarrow k$, even though $\left[S_{\alpha}^{\mu}, S_{\alpha}^{\nu}\right] \neq 0$; indeed (4.20) has this property.

We return to consider matrix elements between $p$ sublattice states such as $|\Phi\rangle$ and $\left|\Phi^{\prime}\right\rangle$. To compute the effective Hamiltonian due to $\left(\delta \hat{\mathcal{H}}_{\text {diff }}\right)^{2}$, we will need the operator equivalent of $\left(\mathbf{s}_{i} \cdot \mathbf{s}_{j}\right)\left(\mathbf{s}_{k} \cdot \mathbf{s}_{l}\right)$ (see (4.13)). We give here the representatives of the three possible situations. (All others are equivalent, by permutation of the sublattice indices.)

$$
\text { 1. Case } \alpha(i)=1, \alpha(j)=2, \alpha(k)=3, \alpha(l)=4
$$

$$
\left\langle\phi^{\prime}\left|\left(\mathbf{s}_{i} \cdot \mathbf{s}_{j}\right)\left(\mathbf{s}_{k} \cdot \mathbf{s}_{l}\right)\right| \phi\right\rangle=\left\langle\phi^{\prime}\left|\frac{1}{\tilde{N}^{4}}\left(\mathbf{S}_{1} \cdot \mathbf{S}_{2}\right)\left(\mathbf{S}_{3} \cdot \mathbf{S}_{4}\right)\right| \phi\right\rangle
$$




$$
\begin{aligned}
& \text { 2. Case } \alpha(i)=\alpha(k)=1, \alpha(j)=2, \alpha(l)=3 \\
& \left\langle\phi^{\prime}\left|\left(\mathbf{s}_{i} \cdot \mathbf{s}_{j}\right)\left(\mathbf{s}_{k} \cdot \mathbf{s}_{l}\right)\right| \phi\right\rangle=\left\langle\phi^{\prime}\right|\left\{\frac{s^{2}}{3 \tilde{N}^{2}}\left(\mathbf{S}_{2} \cdot \mathbf{S}_{3}\right)\right. \\
& +2 s G\left(\mathbf{S}_{1}, \mathbf{S}_{2}, \mathbf{S}_{3}\right)+\left[\frac{s}{3 \tilde{N}^{2}}\left(\mathbf{S}_{2} \cdot \mathbf{S}_{3}\right)-G\left(\mathbf{S}_{1}, \mathbf{S}_{2}, \mathbf{S}_{3}\right)\right. \\
& \left.\left.+\frac{i}{2 \tilde{N}^{3}} \mathbf{S}_{1} \cdot\left(\mathbf{S}_{2} \times \mathbf{S}_{3}\right)\right] \delta_{i k}\right\}|\phi\rangle,
\end{aligned}
$$

where

$$
\begin{gathered}
G\left(\mathbf{S}_{1}, \mathbf{S}_{2}, \mathbf{S}_{3}\right)=\frac{1}{\tilde{N}^{3}(2 \tilde{S}-1)}\left[\frac { 1 } { 2 } \left(\left(\mathbf{S}_{1} \cdot \mathbf{S}_{2}\right)\left(\mathbf{S}_{1} \cdot \mathbf{S}_{3}\right)\right.\right. \\
\left.\left.+\left(\mathbf{S}_{1} \cdot \mathbf{S}_{3}\right)\left(\mathbf{S}_{1} \cdot \mathbf{S}_{2}\right)\right)-\frac{1}{3} \tilde{S}(\tilde{S}+1) \mathbf{S}_{2} \cdot \mathbf{S}_{3}\right] \cdot \\
\text { 3. Case } \alpha(i)=\alpha(k)=1, \alpha(j)=\alpha(l)=2 \\
+\left[\frac{1}{3} s^{3}-2 s F\left(\mathbf{s}_{i} \cdot \mathbf{S}_{j}\right)\left(\mathbf{s}_{k} \cdot \mathbf{S}_{l}\right)|\phi\rangle=\left\langle\phi^{\prime}\right|\left\{\frac{1}{3} s^{4}+4 s^{2} F\left(\mathbf{S}_{1}, \mathbf{S}_{2}\right)\right.\right. \\
\left.+\left[\frac{1}{3} s^{2}+F\left(\mathbf{S}_{1}, \mathbf{S}_{2}\right)-\frac{1}{2 \tilde{N}^{2}}\left(\mathbf{S}_{1} \cdot \mathbf{S}_{2}\right)\right]\left(\delta_{i k} \delta_{j l}\right)\right\}|\phi\rangle, \quad(4
\end{gathered}
$$

where,

$$
\begin{aligned}
F\left(\mathbf{S}_{1}, \mathbf{S}_{2}\right)= & \frac{1}{\tilde{N}^{2}(2 \tilde{S}-1)^{2}}\left[\left(\mathbf{S}_{1} \cdot \mathbf{S}_{2}\right)^{2}+\right. \\
& \left.\frac{1}{2}\left(\mathbf{S}_{1} \cdot \mathbf{S}_{2}\right)-\frac{1}{3} \tilde{S}^{2}(\tilde{S}+1)^{2}\right] .
\end{aligned}
$$

These three formulas are obtained from summing over $\mu, \nu$ in $\sum\left(\mathbf{s}_{i}^{\mu} \mathbf{s}_{j}^{\mu}\right)\left(\mathbf{s}_{k}^{\nu} \mathbf{s}_{l}^{\nu}\right)$ and using (4.7) and 4.20) for the operator equivalents of one single spin and the product of two spins on the same sublattice.

\section{E. Summing over the lattice}

With the operator equivalent of $\left(\mathbf{s}_{i} \cdot \mathbf{s}_{j}\right)\left(\mathbf{s}_{k} \cdot \mathbf{s}_{l}\right)$ we can sum over the lattice and obtain the operator equivalent of $\hat{\mathcal{O}}_{m n}$ in 4.13 ). The results depend on how the sublattices are related geometrically to each other, via $z_{\alpha \beta}^{m}$ defined in Sec. II. Again the representatives of the three possible situations are the following.

$$
\text { 1. Case } \alpha(i)=1, \alpha(j)=2, \alpha(k)=3, \alpha(l)=4
$$

Here we have $\tilde{N} z_{12}^{m}$ pair of $(\alpha(i)=1, \alpha(j)=2)$ and $\tilde{N} z_{34}^{m}$ pair of $(\alpha(k)=3, \alpha(l)=4)$ in the double sum $\sum_{m-\text { th }} \sum_{n-\text { th }}$. The contribution of 4.21 to the total sum is

$$
\frac{1}{\tilde{N}^{2}} z_{12}^{m} z_{34}^{m}\left(\mathbf{S}_{1} \cdot \mathbf{S}_{2}\right)\left(\mathbf{S}_{3} \cdot \mathbf{S}_{4}\right)
$$

$$
\text { 2. Case } \alpha(i)=\alpha(k)=1, \alpha(j)=2, \alpha(l)=3
$$

$\tilde{N} z_{12}^{m} \tilde{N} z_{13}^{m}$ terms and $\tilde{N} z_{12}^{m} z_{13}^{m}$ of them have $i=k$ (for the $\delta_{i k}$ terms in (4.22)). The contribution to the total sum turns out to be simple

$$
\frac{1}{\tilde{N}^{2}} z_{12}^{m} z_{13}^{m}\left(\mathbf{S}_{1} \cdot \mathbf{S}_{2}\right)\left(\mathbf{S}_{1} \cdot \mathbf{S}_{3}\right) \text {. }
$$

We can obtain this result either from the complicated operator equivalent (4.22) or from the fact that here the double sums $\sum_{m-\text { th }} \sum_{n-\text { th }}$ can be summed separately to give the sublattice spins.

$$
\text { 3. Case } \alpha(i)=\alpha(k)=1, \alpha(j)=\alpha(l)=2
$$

This is the most complicated case. There are $\tilde{N} z_{12}^{m} \tilde{N} z_{12}^{m}$ terms and $\tilde{N} z_{12}^{m} z_{12}^{m}$ of them have $i=k$ (for the $\delta_{i k}$ term in (4.24), $\tilde{N} z_{12}^{m} z_{21}^{m}$ of them have $j=l$ (for $\delta_{i k}$ term), and $N z_{12}^{m}$ of them have $i=k$ and $j=l$ (for $\delta_{i k} \delta_{j l}$ term). Here the contribution to the total sum is

$$
\begin{aligned}
& \frac{1}{\tilde{N}^{2}}\left(z_{12}^{m}\right)^{2}\left(\mathbf{S}_{1} \cdot \mathbf{S}_{2}\right)^{2} \\
& -\frac{1}{\tilde{N}^{2}}\left(z_{12}^{m}\right)^{2}\left[\frac{1}{(2 \tilde{S}-1)^{2}}\left(\mathbf{S}_{1} \cdot \mathbf{S}_{2}\right)^{2}-\frac{1}{2}\left(1-\frac{1}{(2 \tilde{S}-1)^{2}}\right)\right. \\
& \left.\left(\mathbf{S}_{1} \cdot \mathbf{S}_{2}\right)+\frac{\tilde{S}^{3}(\tilde{S}-2)}{(2 \tilde{S}-1)^{2}}\right] \\
& +\delta_{m n} \tilde{N} z_{12}^{m}\left(\frac{1}{3} s^{2}+\tilde{N} F\left(\mathbf{S}_{1}, \mathbf{S}_{2}\right)-\frac{1}{2 \tilde{N}^{2}}\left(\mathbf{S}_{1} \cdot \mathbf{S}_{2}\right)\right),
\end{aligned}
$$

where $F$ is given above in 4.25 .

\section{F. Final form of effective Hamiltonian}

Combining the three terms $4.26,4.27,4.28$ ) and their cyclic permutations, we get

$$
\begin{aligned}
& \left\langle\phi^{\prime}\left|\hat{\mathcal{O}}_{m n}\right| \phi\right\rangle=\left\langle\phi^{\prime}\right| \frac{2}{\tilde{N}^{2}(2 \tilde{S}-1)^{2}} \sum_{\alpha \neq \beta}\left[z_{\alpha \beta}^{m}\left(\tilde{N} \delta_{m n}-z_{\alpha \beta}^{n}\right)\right. \\
& \left.\left(\left(\mathbf{S}_{\alpha} \cdot \mathbf{S}_{\beta}\right)^{2}-2 \tilde{S}(\tilde{S}-1)\left(\mathbf{S}_{\alpha} \cdot \mathbf{S}_{\beta}\right)+\tilde{S}^{3}(\tilde{S}-2)\right)\right]|\phi\rangle
\end{aligned}
$$

Finally, we perform $\sum_{m n}$ in (4.12) and use 4.11) and the fact that a singlet state $|\Phi\rangle$ is simply a linear combination of $p$-sublattice states $|\phi\rangle$ to obtain our result for the biquadratic effective Hamiltonian,

$$
\begin{aligned}
\hat{\mathcal{H}}_{\mathrm{eff}} & =-\frac{K_{12}}{4 \tilde{J}_{S} \tilde{N} \tilde{S}(2 \tilde{S}-1)^{2}} \sum_{\alpha \neq \beta}\left[\left(\mathbf{S}_{\alpha} \cdot \mathbf{S}_{\beta}\right)^{2}\right. \\
& \left.-2 \tilde{S}(\tilde{S}-1)\left(\mathbf{S}_{\alpha} \cdot \mathbf{S}_{\beta}\right)+\tilde{S}^{3}(\tilde{S}-2)\right]
\end{aligned}
$$

where 


$$
K_{\alpha \beta}=\sum_{m}\left(J_{m}\right)^{2} z_{\alpha \beta}^{m}-\frac{1}{\tilde{N}}\left(\sum_{m} J_{m} z_{\alpha \beta}^{m}\right)^{2},
$$

and we have used permutation symmetry $K_{\alpha \beta}=K_{12}$. ( $\tilde{N}$ and $z_{\alpha \beta}^{m}$ are defined in Sec. [I. $)$ The middle term of (4.30) can be simplified, since it is linear in $\hat{\mathcal{H}}_{\mathrm{MF}}$ which has the same value for any of the $p$-sublattice singlets that we are considering. The final result is

$$
\hat{\mathcal{H}}_{\mathrm{eff}}\left(\left\{\mathbf{S}_{\alpha}\right\}\right)=-\tilde{K} \sum_{\alpha<\beta}\left(\mathbf{S}_{\alpha} \cdot \mathbf{S}_{\beta}\right)^{2}+\tilde{C},
$$

where

$$
\tilde{K}=\frac{K_{12}}{2 \tilde{J}_{S} \tilde{N} \tilde{S}(2 \tilde{S}-1)^{2}}
$$

and

$$
\tilde{C}=-\frac{1}{2} p \tilde{K} \tilde{S}^{2}\left((p+1) \tilde{S}^{2}-2(p-1) \tilde{S}-2\right) .
$$

By recalling that $\tilde{J}_{S} \sim N^{-1}$ (see eq. (3.5)) and that $\mathbf{S}_{\alpha} \sim$ $\tilde{S}=N s / p$, it may be checked that (4.32) scales with $N$.

In the limit $N \rightarrow \infty$, the operator $\mathbf{S}_{\alpha}$ becomes perfectly defined and can be equated to a c-number, $\tilde{S} \hat{\mathbf{m}}_{\alpha}$, where $\hat{\mathbf{m}}_{\alpha}$ is a unit vector denoting the classical direction of sublattice $\alpha$. Then our effective Hamiltonian (4.32) simplifies further to

$$
-\frac{\left(\sum_{m} J_{m}^{2} z_{12}^{m}\right) s}{8\left(\tilde{J}_{12}-\tilde{J}_{11}\right)}\left(\sum_{\alpha<\beta}\left(\hat{\mathbf{m}}_{\alpha} \cdot \hat{\mathbf{m}}_{\beta}\right)^{2}+\frac{1}{2} p(p+1)\right) .
$$

Earlier, Larson and Henley order effective Hamiltonian starting from a different sort of mean-field theory:

$$
\Delta \mathcal{H}=-\frac{s^{2}}{8 h_{0}} \sum_{\langle i j\rangle} J_{i j}^{2}\left(1-\hat{\mathbf{m}}_{i} \cdot \hat{\mathbf{m}}_{j}\right)^{2} .
$$

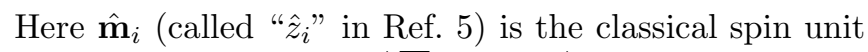
vector at site $i$ and $h_{0}=\left|\sum_{j} J_{i j} s \hat{\mathbf{m}}_{j}\right|$ is the magnitude of the local field at $i$. Their result is valid for Hamiltonians more general than the " $p$-sublattice" category, on the other hand it is valid only in infinite systems. In the special case that sites are grouped into $p$ sublattices, as assumed in this paper, it can be checked that (4.36) does reduce to our effective Hamiltonian (4.35) for the $N \rightarrow \infty$ limit, with $h_{0}=\left(\tilde{J}_{12}-\tilde{J}_{11}\right) \tilde{N} s$.

\section{SEMICLASSICAL CALCULATION OF SINGLET ENERGIES}

The effective biquadratic Hamiltonian (4.32) is the basis for an analytic approximation of the energy eigenvalues for a $p=4$ spin antiferromagnet, as detailed in Ref. 1.
A further series of mappings is applied to the effective Hamiltonian resulting in a semiclassical problem of one spin with a quartic Hamiltonian. Bohr-Sommerfeld quantization and tunneling considerations are then applied to this one-spin problem to obtain energies and level splittings. The procedure here is identical to that in Ref. 1 which worked out the triangular lattice case, except that the coefficients are different, being functions of $J_{1}$ and $J_{2}$ as well as the type and size of the lattice.

This section recapitulates and somewhat extends the recent calculation by two of ust of the low-lying singlet energies of the four-sublattice Heisenberg antiferromagnets, assuming long-range order in the $N \rightarrow \infty$ limit. That derivation started from a four-spin system with the biquadratic effective Hamiltonian (4.32) derived above. When the total spin is zero, as is the case here, the classical dynamics is separable into a trivial and a nontrivial part (Sec. V A). The nontrivial part is very similartoa single spin in an anisotropy field of cubic symmetry.20 22

Next, using Bohr-Sommerfeld quantization of the classical orbits of this effective one-spin Hamiltonian, the low-lying singlet energies were shown to form clusters of two or three degenerate levels and the energies were approximately estimated (Sec. VB). It was found that the lower energy clusters are threefold degenerate, corresponding to excitations near the "collinear" states of the classical ground state manifold (with four spins forming two pairs that point in opposite directions), and the higher energy clusters are twofold degenerate, corresponding to noncollinear "tetrahedral" states (with four spins pointing at the four vertices of a tetrahedron).

Finally, tunneling between Bohr-Sommerfeld orbits in different energy wells generates splittings exponentially small (in the cluster size) within the level clusters, except when there is destructive interference of tunneling amplitudes associated with different paths which leaves the clusters unsplit. From the geometrical phase of the tunneling paths, 23 one could predict the pattern of split and unsplit states in the clusters (Sec. VC). The predicted pattern of level clustering was in accord with numerical datal 2 for the $J_{1}-J_{2}$ triangular lattice with $N=16$ and $N=28$.

We add a new discussion of the other quantum numbers, derived from the spatial symmetries of the spin arrangements (Sec. VD). Since it is advantageous for exact diagonalizations to break the Hamiltonian into blocks with different symmetries, the symmetry eigenvalues are often obtained as a byproduct, which may now be checked against our predictions.

The section concludes (Sec. VE) with a discussion of the system-size dependences of the various energy scales characterizing low-lying eigenstates. This illustrates how brute-force diagonalization of a (necessarily small) system can be misleading as to the energy scales of a large system, and how an analytic view can correct this. 


\section{A. Coherent states and separation of rotational variables}

We now carry out the semiclassical calculation on our quantum $p$-spin effective Hamiltonian (4.32) as in Ref. 1. Given a quantum spin Hamiltonian, we can obtain upper and lower bounds of the quantum partition function using the classical integral representation.24 A classical spin Hamiltonian can then be derived and is the starting point of semiclassical calculations. 25 We note there are multiple ways to define this semiclassical spin Hamiltonian and two of which correspond to the upper and lower bounds of the partition function respectively (they are called $\mathcal{H}_{Q}$ and $\mathcal{H}_{P}$ respectively in Ref. 25). Here we will use the $\mathcal{H}_{Q}$ option which is obtained by taking expectations of the quantum Hamiltonian within spin coherent states.

Using spin coherent states $\left|\left\{\hat{\mathbf{m}}_{\alpha}\right\}\right\rangle$ where $\hat{\mathbf{m}}_{\alpha}$ is a unit vector (see, e.g., Ref. 26), with $1 \leq \alpha \leq p$, we get,

$$
\begin{aligned}
& \mathcal{H}_{\mathrm{eff}}\left(\left\{\hat{\mathbf{m}}_{\alpha}\right\}\right) \equiv\left\langle\left\{\hat{\mathbf{m}}_{\alpha}\right\}\left|\hat{\mathcal{H}}_{\mathrm{eff}}\right|\left\{\hat{\mathbf{m}}_{\alpha}\right\}\right\rangle \\
& =-K_{U} \sum_{\alpha<\beta}\left(\hat{\mathbf{m}}_{\alpha} \cdot \hat{\mathbf{m}}_{\beta}\right)^{2}+C_{U},
\end{aligned}
$$

where $K_{U}=\tilde{K} \tilde{S}^{2}(\tilde{S}-1 / 2)^{2}$ and $C_{U}=-p \tilde{K} \tilde{S}^{2}(4 \tilde{S}(p-$ 1) $+p+1) / 8+\tilde{C}$. For a closed orbit in this space $\left\{\hat{\mathbf{m}}_{\alpha}(t)\right\}$ parametrized by $t$, the geometrical phase (important for semiclassical calculation) is,

$$
\Phi=\sum_{\alpha=1}^{4} \tilde{S} \Omega\left(\left\{\hat{\mathbf{m}}_{\alpha}(t)\right\}\right)
$$

where $\Omega(\{\hat{\mathbf{m}}(t)\})$ denotes the spherical area the trajectory $\hat{\mathbf{m}}(t)$ has swept out on the unit sphere around the $z$ axis. Because each of the $p$ unit vectors lives on a two-dimensional spherical surface, so with the constraint $\sum \hat{\mathbf{m}}_{\alpha}=0$, we have a $(2 p-3)$-dimensional space specified by $\left\{\hat{\mathbf{m}}_{\alpha}\right\}$. Furthermore, the global rotation separates from the internal rotations (at least for $p=4$, 1 this depends only on the permutation symmetry of eq. (4.32) ). We are left with a simpler problem on a two-dimensional spherical surface specified by a new unit vector $\hat{\mathbf{n}}$.

From now on, we will work on the special case $p=4$ which is the case for the fcc and triangular lattices. First, let $\mathbf{n}_{\mu}=\left(\hat{\mathbf{m}}_{\mu}+\hat{\mathbf{m}}_{4}\right) / 2, \mu=1,2,3$ (not a unit vector). It is easy to check that these three vectors are mutually orthogonal; therefore, given a coordinate system $\left\{\hat{\mathbf{e}}_{\mu}\right\}$, we can rotate $\left\{\mathbf{n}_{\mu}\right\}$, with a proper rotation matrix $\mathcal{R}$, so that $\mathcal{R} \mathbf{n}_{\mu}=n_{\mu} \hat{\mathbf{e}}_{\mu}$, where $n_{\mu}$ are scalar coefficients. We then form a new vector $\hat{\mathbf{n}}=\left(n_{1}, n_{2}, n_{3}\right)$; it is easy to check that this is a unit vector $\left(\mathbf{n}_{1}^{2}+\mathbf{n}_{2}^{2}+\mathbf{n}_{3}^{2}=1\right)$. In terms of this $\hat{\mathbf{n}}$, we obtain a one-spin semiclassical Hamiltonian from (5.1),

$$
\mathcal{H}_{1}(\hat{\mathbf{n}})=-8 K_{U} \sum_{\mu=1}^{3} n_{\mu}^{4}+2 K_{U}+C_{U} .
$$

It is easy to show that $\mathcal{R} \hat{\mathbf{m}}_{1}=\left(n_{1},-n_{2},-n_{3}\right)$, $\mathcal{R} \hat{\mathbf{m}}_{2}=\left(-n_{1}, n_{2},-n_{3}\right), \mathcal{R} \hat{\mathbf{m}}_{3}=\left(-n_{1},-n_{2}, n_{3}\right), \mathcal{R} \hat{\mathbf{m}}_{4}=$ $\left(n_{1}, n_{2}, n_{3}\right)$, which says that each $\hat{\mathbf{m}}_{\alpha}(t)$ traces out the same shaped trajectory as $\hat{\mathbf{n}}(t)$ and makes the same contributation to the geometrical phase (5.2). Therefore, we have $\Phi=4 \tilde{S} \Omega(\hat{\mathbf{n}}(t))=S \Omega(\hat{\mathbf{n}}(t))$, where we have used $S=4 \tilde{S}=N s$. In the sense of semiclassical orbits and geometrical phases, we have mapped the problem of Hamiltonian (5.1) with four spins $\tilde{S}$ to the problem of Hamiltonian (5.3) with one spin $S$.

It must be pointed out that the definition of our coherent states $\left|\left\{\hat{\mathbf{m}}_{\alpha}\right\}\right\rangle$ included an averaging over all rotations. It follows that the mapping to $\hat{\mathbf{n}}$ is not one-to-one. If $\mathcal{R}_{\pi}$ is a $\pi$-rotation about any coordinate axis $\hat{\mathbf{e}}_{\alpha}$, then $\left(\mathcal{R}_{\pi} \mathcal{R}\right) \mathbf{n}_{\mu}=\mathbf{n}_{\mu}^{\prime} \hat{\mathbf{e}}_{\mu}$, and so the rotation matrix $\mathcal{R}_{\pi} \mathcal{R}$ satisfies our condition just as well as $\mathcal{R}$. In other words, in the $\hat{\mathbf{n}}$ representation, two vectors related by a $\pi$ rotation about any coordinate axis represent the same four-spin state $\left\{\hat{\mathbf{m}}_{\alpha}\right\}$. We need to take this discrete redundancy into account in the semiclassical calculation below. 27

\section{B. EBK quantization}

To obtain energies from the semiclassical spin Hamiltonian, we use the EBK, or Bohr-Sommerfeld (BS), quantization condition which says that the geometrical phase of the Bohr-Sommerfeld orbit is $2 \pi l$ where $l=0,1, \ldots, 2 S$. 28 (The BS semiclassical calculation described in this section was carried out in Ref. 1 for the triangular system. The difference here for the fcc system is the value of $S$.) On the sphere, the spherical angle enclosed by two curves $\theta_{1}(\phi)$ and $\theta_{2}(\phi)$ is $\int\left(\cos \theta_{1}-\cos \theta_{2}\right) d \phi$, where the integration limits are determined by the geometrical locations of the two curves. For our quartic Hamiltonian, we first consider

$$
u=n_{1}^{4}+n_{2}^{4}+n_{3}^{4}=x^{2}+(1-x)^{2} a(\phi),
$$

where $x=\cos ^{2} \theta$ and $a(\phi)=\cos ^{4} \phi+\sin ^{4} \phi=(3+$ $\cos 4 \phi) / 4$. Solving this quadratic equation, we get,

$$
\cos \theta= \pm \sqrt{\frac{a \pm \sqrt{u-(1-u) a}}{a+1}},
$$

where the \pm signs need to be chosen according to the location of the orbit.

As mentioned in Ref. 1, two types of orbits on the $\hat{\mathbf{n}}$ unit sphere are relevant. (1) "Collinear" (C) states that orbit around the three coordinate axes (they are therefore three-fold degenerate). The quantization condition for these states is $\Phi=4 \pi l$ (a factor of two is present because of the rotation by $\pi$ redundancy mentioned above). (2) "Tetrahedral" ( $\mathrm{T}$ ) states that orbit around two axes $( \pm 1,1,1) / \sqrt{3}$ (and equivalents) (they are then two-fold degenerate). Here the quantization condition is $\Phi=2 \pi l$.

For the $C$-type orbits, the BS quantization condition leads to the equation, 
$\Omega_{C_{l}}=\int_{0}^{2 \pi}\left(1-\sqrt{\frac{a(\phi)+\sqrt{u-(1-u) a(\phi)}}{a(\phi)+1}}\right) d \phi=\frac{4 \pi l}{S}$.

For $T$-type orbits, we get,

$$
\begin{aligned}
\Omega_{T_{l}}= & 2 \int_{1 / 2}^{u /(1-u)}\left[\sqrt{\frac{a+\sqrt{u-(1-u) a}}{a+1}}\right. \\
& \left.-\sqrt{\frac{a-\sqrt{u-(1-u) a}}{a+1}}\right] \frac{d a}{\sqrt{1-(4 a-3)^{2}}}=\frac{2 \pi l}{S},
\end{aligned}
$$

where in the last integral we have changed integration variable to $a$, using $d \phi= \pm d a / \sqrt{1-(4 a-3)^{2}}$ ( \pm signs determined by location). Without loss of generality, the $C$-orbit equation is obtained from orbits around $(0,0,1)$ and the $T$-orbit equation from those around $(1,1,1) / \sqrt{3}$. For given $S$ and $l$, these two equations are solved numerically for $u$ using Mathematica, and the semiclassical energy is then $K_{U}(2-8 u)+C_{U}$. Semiclassical energies are presented in Tables III and IV for fcc and triangular lattices respectively. The result for the triangular case corrects a mistake in the last column of Table I in Ref. 1 which came from a wrong sign in the expression for $C_{U}$ just under eq. (6) in Ref. 1. The correct expression should be $C_{U}=-\tilde{S}^{2}(5 \tilde{S}+5 / 2) K+\tilde{C}_{\text {biq }}$.

\section{Tunnel splittings and eigenvalue fine structure}

The preceding section establishes that the low-lying levels are grouped into clusters of three in the lower part (corresponding to the three-fold degenerate $C$-type orbits) and clusters of two in the higher part (corresponding to the two-fold degenerate $T$-type orbits). Average energies for the clusters have been calculated from the BS quantization condition. Finer level splittings within each cluster are results of tunneling between BS orbits, and degeneracies can remain due to destructive interfer ence of the geometrical phases of the tunneling paths. 23 Using phase considerations, we can therefore obtain the split/unsplit pattern for energy clusters: it depends on $N, s, l$.

We here simply quote the result from Ref. 1. If we write " $\left(\nu_{1}, \nu_{2}\right)$ " for the pattern of eigenvalues in a cluster, meaning that the (lower,higher) eigenvalues have degeneracies $\left(\nu_{1}, \nu_{2}\right)$, respectively, then for the three-fold degenerate $C$-type orbits, we obtain a cluster pattern $(2,1)$ if the expression $N s / 2-3 l$ is odd or $(1,2)$ if it is even; and for the two-fold degenerate $T$-type orbits, we get $(1,1)$ when $N s-4 l$ is divisible by three, or (2), i.e., unsplit, otherwise.29 Notice that, ignoring whether the splittings are large or small, the pattern is $11221122 \ldots$ at either end. Indeed this sequence continues unbroken across the energy of the classical separatrix of the orbits, which is the boundary between the two clustering behaviors (i.e., where the $C$-type orbits meet the $T$-type). As energy increases, the splitting within each cluster grows, until at the separatrix energy $(u=1 / 2)$ it is typically comparable to the separation between successive clusters, causing an ambiguity in labeling the levels.

The case of fcc type-I with $N=16$ is trivial since $\delta \hat{\mathcal{H}} \equiv 0$ which means that the four-sublattice singlets are the exact ground states, and are exactly degenerate. Our calculation, of course, gets this right since it gives a zero coefficient in the effective Hamiltonian $\left(K_{12}=\right.$ $4(\tilde{N}-4) J_{1}^{2} / \tilde{N}$ and $\left.\tilde{N}=4\right) .30$ Here, $\tilde{S}=2$ and we have 5.7) $2 \tilde{S}+1=5$ degenerate singlet states (see Appendix A).

Table III shows the results For fcc Type-I with $N=$ $32, s=1 / 2$. The preceding semiclassical considerations lead to an energy pattern which begins with $(1,2)(2,1)$ and ends with $(1,1)(2)$. These clusters correspond to orbits $C_{0}, C_{1}, T_{1}$, and $T_{0}$. The total pattern could be written as $(1,2)(2,1 / 1,1)(2)$, where the " $1 / 1$ " indicates a single level which can equally well be assigned as the highest level in cluster $C_{1}$ or the lower level of cluster $T_{1}$. In Table III], the entries with "..." correspond to this level with the ambiguous labeling. The diagonalization results (especially for the full 32-site system, see Sec. VI G below) illustrate how, near the separatrix energy, the inter- and intra-cluster splittings become comparable.

Note that in this paper we have not attempted to estimate the tunnel splitting (i.e., the splitting within each cluster) quantitatively, because we have only computed the "WKB" exponent in this splitting and not its prefactor (all these splittings are exponential in cluster size) 1,1132

\section{Spatial symmetries}

The Hamiltonian (1.1) is invariant under the spacegroup $\mathcal{L}$, which acts on the lattice of spins. Consequently the eigenstates transform as representations of this symmetry group; in particular, they all (can be chosen to) have definite crystal momentum under lattice translations. Similarly, our $p$-spin Hamiltonian (4.32) is invariant under all permutations of the $p$ spins, so its eigenstates transform as the representations of the permutation group $\mathcal{S}_{p}$.

Now, as observed by Lecheminant et al, $\mathrm{l}$ each spacegroup operation induces a permutation of the $p$ sublattices. Thus the symmetry eigenvalues of the $p$-spin eigenstates under the permutation group are the same as those of the corresponding eigenstates of the real Hamiltonian under the corresponding space operation. Ref. 2 combined this idea with group theory to count the number of times each representation appears among the $p$-spin singlets. Here, we outline how the same notion may be combined with semiclassics to identify which level has which quantum number, including the specific correspondence for both the triangular and fcc realizations of $p=4$. That will require an additional mapping from $p$-spin per- 
mutations to (certain) cubic-symmetry point operations on the $\mathbf{n}$-vector in (5.3).

Our $p$-spin approximation groups the $\tilde{N}$ spins of one sublattice into one big spin of maximum length, i.e. our states are totally symmetric under any permutation of sites within a sublattice. Thus we implicitly assumed that the symmetry eigenvalue is unity under any translation that takes spins into the same sublattice. In effect, such translations are equivalenced to the identity, and the entire space group reduces to $\mathcal{S}_{p}$ (or a subgroup).

In the four-sublattice case, every lattice translation is equivalent (if not to the identity) to a permutation of the class $(12)(34)$. (We write permutations in the standard notation where each parenthesis is a cyclic permutation and each number is the label of a sublattice.) But for any configuration of the four classical directions $\left\{\hat{\mathbf{m}}_{\alpha}\right\}$, that permutation can be implemented instead by a $\pi$-rotation about the axis $\mathbf{n}_{3}$. Since we defined our coherent state $\left|\left\{\hat{\mathbf{m}}_{\alpha}\right\}\right\rangle$ to be symmetrized with respect to all rotations, it follows that the state pust be even under permutations of the $(12)(34)$ class.33 One corollary is that, in the 4spin case, the low-lying singlets all have wavevector zero, as indeed was found in the exact diagonalizations of the triangularla and of the fcc type I (Sec. VIC).

Another corollary is that all permutations of this class are equivalenced to the identity; in effect, our permutation group is replaced by $\mathcal{S}_{4} / \mathcal{Q} \cong \mathcal{S}_{3}$ where $\mathcal{Q}$ is the subgroup consisting of $(12)(34),(13)(24),(14)(23)$ and the identity.

The permutations of class (123) correspond to the threefold axes in the triangular lattice or the fcc lattice, as well as about the threefold axes $( \pm 1,1,1) / \sqrt{3}$ of the n-sphere under Hamiltonian (5.3). Group theory suffices to identify the behavior of each state under (123): the nondegenerate states have eigenvalue 1 and the twofold degenerate states have eigenvalues $e^{ \pm 2 \pi i / 3}$.

However, group theory is insufficient to identify the eigenvalues for the nondegenerate states under the odd permutations, which might be +1 or -1 . The permutations of class (12) correspond to any lattice reflection in the triangular lattice, or reflections in a $\{110\}$-type plane for the fcc lattice. The permutations of class (1234) correspond to glide planes in either lattice, but they are equivalent to the (12) class modulo subgroup $\mathcal{Q}$. On the $\mathbf{n}$-sphere, an odd permutation is best represented by a $\pi / 2$-rotations about one of the three coordinate axes. (Recall that the $\pi$-rotations are equivalenced to the identity.)

Consider the eigenstates in a three-cluster, mixed from three BS orbits $C_{l X}, C_{l Y}$, and $C_{l Z}$ (these are collinear states rotating around the $X, Y$, and $Z$ axes respectively). The wavefunction described by $C_{l X}$ picks up a phase $4 \pi l$ when it goes full circle, 1 thus it gets multipied by $e^{i \pi l}=(-1)^{l}$ under a $\pi / 2$ rotation about the $X$ axis (that orbits center). The nondegenerate eigenstate has an equal admixture of all three states, in particular $C_{l X}$, so it must have eigenvalue $(-1)^{l}$ under this rotation, or equivalently under the permutation (23).34 Thus, the lone eigenstate in the lowest cluster is even under odd permutations of sublattices, and alternates odd/even from then on. The twofold degenerate eigenstate contains can be broken into one odd and one even state (the state with eigenvalue $-(-1)^{l}$ under $(23)$ is a mixture only of $C_{l Y}$ and $C_{l Z}$ ).

Numerical diagonalization of the four-spin system (for all $\widetilde{S} \leq 13 / 2$ ) confirms the predicted pattern of even/odd states (see Sec. VIA). In the exact-diagonalization results, it can only be checked in the case of the $N=$ 16 triangular lattice (which agrees) 35 Empirically, the even/odd alternation (of the nondegenerate states) continues across the separatrix to the higher-energy clusters composed of two states, which are mixed from the two BS orbits called $T_{l \pm}$. It turns out that whenever such a pair is split, the lower level is even (odd) and the upper level is odd (even) under the odd permutations, according to whether $l$ is even (odd).

\section{E. Dependence on size $N$}

How do our results relate to the macroscopic limit $N \rightarrow \infty$ ? It has long been understood 36 that in quantum antiferromagnets symmetry-broken states (those with a nonzero expectation of each spin) are superpositions of a family of eigenstates which become degenerate with the ground state only as $N \rightarrow \infty$. This motivates a classification of the smallest energy gaps, measured from the ground state.

In the present case of a four-sublattice system, the smallest gap is within a cluster of three singlets (as derived in Ref. 11) which are mixed from the lowest Bohr-Sommerfeld orbits $\left(C_{X 0}, C_{Y 0}, C_{Z 0}\right)$ as discussed in Sec. VB As discussed above, this gap is a tunnel splitting 37 and is exponentially small in $N$. (The same is true for any discrete symmetry breaking. But this gap is larger than in many other cases, since the tunnel barrier comes entirely from quantum fluctuations.)

The other gaps are associated in a well-known fashion with the continuous symmetries or degeneracies of the classical system. If a continuous symmetry is broken and the interactions are short-range, there are always gapless "Goldstone mode" excitations, in this case the acoustic magnons. The states with one long-wavelength magnon (at a nonzero wavevector $\mathbf{k}$ ) have much larger energies, $E=O\left(1 / N^{1 / d}\right)$ as $N \rightarrow \infty$ in a $d$-dimensional system. This follows from the antiferromagnetic magnon dispersion $\omega=O(|\mathbf{k}|)$ and from $|\mathbf{k}|=O\left(1 / N^{1 / d}\right)$ for the smallest wavevector (with periodic boundary conditions). Our present theory does not address the magnon states, and their gap is not so small anyhow in a finite system.

The limit of the acoustic magnon as $\mathbf{k}=0$ has zero energy, but only in the $N \rightarrow \infty$ limit. It is better to view it from the classical formula $E=M^{2} / 2 N \chi$ where $M$ is the total magnetization and $\chi$ is the susceptibility (per 
spin) in the macroscopic limit. Then provided $S_{\text {tot }} / N \ll$ 1, the quantum state of total spin $S_{\text {tot }}$ has energy $E=$ $S_{\text {tot }}\left(S_{\text {tot }}+1\right) / 2 N \chi$. This "tower" of multiplets is generic whenever the system breaks spinretation symmetry and the ground state is a singlet.36.38.39 These states have total wavevector 0 , but $S_{\text {tot }}>0$. Their gap is much smaller than the magnons'; it is the next-smallest kind of gap after the tunnel splitting. lowest state of spin 1 .

Finally, just as the rotational symmetry breaking implies the Goldstone modes, the additional continuous degeneracy of our four-sublattice classical ground states implies a gapless "degeneracy mode" at harmonic order, with $\omega_{\text {degen }}(\mathbf{k}=0)=0$. However, quantum fluctuations - approximated by our biquadratic effective Hamiltonian (4.32) - break that degeneracy, creating a gap 40,41 $\omega_{\text {degen }}(\mathbf{k}=0)=\Delta$ this gap becomes constant as $N \rightarrow \infty$.

On the other hand, $\Delta$ is exactly the energy spacing between successive Bohr-Sommerfeld orbits surrounding a single collinear state on the $\mathbf{n}$-sphere (orbits called " $C_{l X}$ " in Ref. 1). It can be verified that our semiclassical prescription indeed implies a constant limiting $\Delta$ in the large $N$ limit. Expanding the "one-spin" effective Hamiltonian (5.3) about a minimum point (e.g. the north pole), the $l$-th orbit has energy $U_{l}=U_{0}+16 K_{U} \theta_{l}^{2}$, where $\theta_{l}$ is the radius of that (nearly circular) orbit on the unit sphere (assuming $S=N s$ is large enough that $\theta_{l} \ll 1$ ). Furthermore the orbit area is $\Omega_{l}=\pi \theta_{l}^{2}$, so from the BS quantization condition (5.6) we obtain $U_{l}=U_{0}+l \Delta$ where the gap $\Delta=64\left(K_{U} / S\right)$.

At this point we can trace back the $N$ dependence of the parameters in the successive versions into which the Hamiltonian has been mapped (see Table III). Writing " $J$ " to schematically represent all couplings, and " $z$ " to represent the total coordination number, we get $\tilde{J}_{S}=O(J z / \tilde{N}), K_{12}=O\left(J^{2} z\right), \tilde{K}=O\left(J / 8 \tilde{S}^{3}\right)$, and $K_{U}=O(J \tilde{S} / 8)=O(J S / 32)$. Thus finally, $\Delta=O(2 J)$, constant to leading order in $1 / N$.

The importance of the above discussion is that at $N=16,28$, or 32 , the apparent $\Delta$ is still strongly sizedependent, and is smaller than the "tower" gap to the first eigenstate with spin $M=1$. Only the analytic analysis reveals that the latter energy scale will become smaller in a large enough system.

\section{NUMERICAL RESULTS FOR FCC TYPE I}

In this section, we present our numerical results from exact diagonalization for a lattice with various levels of approximation based on our effective $p$-spin Hamiltonian (4.32). First, we diagonalize it directly for $p=4$ with parameters suitable for the fcc Type I system. Then we will compare the predictions from four-spin diagonalization and semiclassics with an exact diagonalization of a 32-site system. (The exact diagonalization of a 16-site fcc Type I system gives five degenerate singlet states which are what we expect from theory (see Sec. VC).) Finally, we will also give numerical results for $N=16$ and $N=28$ triangular lattices, correcting some minor numerical mistakes in Ref. 11.

\section{A. Diagonalization of a four-spin system}

We have exactly diagonalized the four-spin Hamiltonian (4.32), as in Ref. 1, representing a fcc lattice with $N=32, s=1 / 2(\tilde{N}=8, \tilde{S}=4)$. As discussed in Ref. 42 , the classical antiferromagnetic Type I ordering occurs when $J_{1}>0, J_{2}<0$. Here we have used $J_{1}=1, J_{2}=-1$. Our code was previously described in Ref. 10. In the diagonalization here, we have used the conservation of total magnetization in the $z$ direction, $S_{\text {tot }}^{z}$, to reduce the size of the Hamiltonian matrix, and also the (1234) cyclic permutation symmetry among the four spins. (See the discussion of odd permutations in Sec. VD.) We identify the singlet states as those which appear in the sector with total $z$ spin 0 , but not in the sector with total $z$ spin 1 .

The result is presented in Table III (and in Table IV for the triangular case). It should be emphasized that this calculation may be done for systems much larger than $N=32$. We could handle $\tilde{S}=30$ which corresponds to $N=240$ for a spin $1 / 2, p=4$ system.

\section{B. Comparison to a one-spin Hamiltonian}

As we mentioned above, there are multiple ways to get a semiclassical spin Hamiltonian from a given quantum spin Hamiltonian. Different prescriptions agree to the leading order in spin, but the higher order corrections are much harder to obtain. To estimate the error of our semiclassical Hamiltonians $\mathcal{H}_{\text {eff }}\left(\left\{\hat{\mathbf{m}}_{\alpha}\right\}\right)$ (5.1) and $\mathcal{H}_{1}(\hat{\mathbf{n}})(5.3)$ with respect to the quantum four-spin Hamiltonian $\hat{\mathcal{H}}_{\text {eff }}\left(\left\{\mathbf{S}_{\alpha}\right\}\right)$ (4.32), we construct a one-spin quantum Hamiltonian $\hat{\mathcal{H}}_{1}(\mathbf{S})$ using spin coherent state $|\hat{\mathbf{n}}\rangle=|\theta, \phi\rangle$, such that $\left\langle\theta, \phi\left|\hat{\mathcal{H}}_{1}(\mathbf{S})\right| \theta, \phi\right\rangle=\mathcal{H}_{1}(\hat{\mathbf{n}})$. (In the notation of Ref. 25, $\hat{\mathcal{H}}_{1}(\mathbf{S})$ is the quantum Hamiltonian for which $\mathcal{H}_{1}(\hat{\mathbf{n}})$ is the $\mathcal{H}_{Q}$ semiclassical Hamiltonian.) $\hat{\mathcal{H}}_{1}(\mathbf{S})$ and $\hat{\mathcal{H}}_{\text {eff }}\left(\left\{\mathbf{S}_{\alpha}\right\}\right)$ then correspond to the same semiclassical Hamiltonian $\mathcal{H}_{1}(\hat{\mathbf{n}})$, and by comparing their eigenvalues we can estimate the error introduced in the semiclassical calculation.

Here we need the expectation value of the quantum operator $\hat{S}_{x}^{4}+\hat{S}_{y}^{4}+\hat{S}_{z}^{4}$ in coherent states. We use the following identity (see Appendix B):

$$
\begin{aligned}
& \left\langle\theta, \phi\left|\hat{S}_{x}^{4}+\hat{S}_{y}^{4}+\hat{S}_{z}^{4}-\frac{3}{5}\left(S^{2}(S+1)^{2}-\frac{1}{3} S(S+1)\right)\right| \theta, \phi\right\rangle \\
= & S\left(S-\frac{1}{2}\right)(S-1)\left(S-\frac{3}{2}\right) \\
& \left(\cos ^{4} \theta+\sin ^{4} \theta \cos ^{4} \phi+\sin ^{4} \theta \sin ^{4} \phi-\frac{3}{5}\right)
\end{aligned}
$$




$$
=\frac{S\left(S-\frac{1}{2}\right)(S-1)\left(S-\frac{3}{2}\right)}{S^{4}}\left(S_{x}^{4}+S_{y}^{4}+S_{z}^{4}-\frac{3}{5} S^{4}\right) .
$$

Here we have emphasized quantum operators with $\hat{S}_{\mu}$, and in the last line, $S_{\mu}$ denotes classical spin variables $\left(S_{\mu}=S n_{\mu}\right.$, where $n_{\mu}$ is defined in (5.3) above). We therefore get a one-spin quantum Hamiltonian from the semiclassical $\mathcal{H}_{1}(\hat{\mathbf{n}})(5.3)$,

$$
\hat{\mathcal{H}}_{1}(\mathbf{S})=-K_{Q} \sum_{\mu=1}^{3} \hat{S}_{\mu}^{4}+C_{Q}
$$

where

$$
K_{Q}=\frac{8 K_{U}}{S(S-1 / 2)(S-1)(S-3 / 2)},
$$

and

$$
C_{Q}=\frac{3}{5}\left(S^{2}(S+1)^{2}-\frac{1}{3} S(S+1)\right) K_{Q}-\frac{14}{5} K_{U}+C_{U} .
$$

Note that here $S=4 \tilde{S}$ is the total spin length.

We have diagonalized this one-spin Hamiltonian (6.2) with $S=16$ for the $N=32, s=1 / 2$ fcc system. It has the same semiclassical approximation as our four-spin system, except that half the eigenstates of the one-spin system are disallowed in the four-spin system. We emphasize that the single-spin system is not a valid model for the extended $N$-spin system in any approximation. It is included only to illustrate the errors in the semiclassical approximations mentioned above, since exact diagonalization gives different results for the one-spin and four-spin quantum cases. Tables [II] and IV contain exact diagonalization results for this Hamiltonian (6.2).

\section{Exact diagonalization of the $N=32$ system}

The lowest-lying states of a cubic 32-spin cluster with $f c c$ structure and periodic boundary conditions were calculated by numerical diagonalization. This is the smallest cluster that can accomodate all three types of antiferromagnetic order predicted by mean field theory (AF-I, AF-II and AF-III), depending on the values of $J_{1}$ and $J_{2}$. It is a consequence of the periodic boundary conditions that for a given site, the six different next-nearest neighbor sites become pairwise identical, such that a spin is only coupled to three different next-nearest neighbor spins, each with the strength $2 J_{2}$. Our calculations show that thisdoes not affect the type of order adopted by the system. 1.43

The size of the diagonalization problem was reduced by employing the following symmetries of the Hamiltonian: total magnetization $S_{z}$, translations, reflections in planes of the $\{100\}$ type, and the simultaneous flipping of all spins; and working in subspaces with particular eigenvalues of these symmetries. The spin-flip symmetry is only useful for $S_{z}=0$ and the reflections are only useful if the translational eigenvalues $e^{i \mathbf{k} \cdot \mathbf{r}}$ correspond to wavevectors $\mathbf{k}=0$ or $\mathbf{k}=(\pi, 0,0)$. For $S_{z}=0$ a subspace dimension of 1213429 was obtained, while for $S_{z}=1$, where the spin-flip symmetry was not used, the dimension was 2259363 .

Fortunately, the discussion in Sec. VD, shows that the predicted low-lying singlets all have $\mathbf{k}=0$ and hence are included in the subspaces we checked. Note also that reflections of the $\{100\}$ type map each sublattice into itself, i.e. they correspond to the identity permutation in $\mathcal{S}_{4}$, so all the low-lying states are predicted to be even under this symmetry, which is confirmed by our results.

The lowest lying eigenstates and eigenenergies were calculated using the Lanczos algorithm 445 with a random seed. If an energy level is degenerate, a single run of the algorithm will only find one eigenvector with this energy. In order to determine the degeneracy of the lowest levels we ran the algorithm several times, each time with a seed that was orthogonal to the low-lying states found in previous runs, so that only the remaining state space was investigated. Bue to numerical instabilities inherent in the algorithm, 45 the orthogonalization was also applied after each step of the Lanczos procedure. The program was tested on a 16-spin $f c c$ cluster for which eigenvalues and degeneracies can be found analytically, and gave correct results. Further details of our method are found in Ref. 9 .

The exact diagonalization results for the fcc lattice are in Table [II]. In Table [V] we also show the results for the triangular case which was diagonalized in Ref. 2, correcting a factor-of-two mistake in Table I of Ref. 11. (The Hamiltonian used in Ref. 2 is twice the Hamiltonian in Ref. 1 and here.)

\section{CONCLUSION}

In summary, we have studied a class of antiferromagnets such that their classical ground states have a $p$ sublattice structure and contain nontrivial degeneracies due to frustration. We are interested in the low-lying energy clustering patterns and have used a $p$-sublattice approach following the structure of the classical ground states. A degenerate family of singlet ground states is introduced, and an effective Hamiltonian is derived to account for the fluctuations within sublattices and is used to calculate the low-lying energies of the antiferromagnet. This effective Hamiltonian couples the sublattice spins and is of the biquadratic form. It is written explicitly for a finite-size lattice and is therefore useful for comparison with exact diagonalization results. We diagonalized a 32site fcc Type-I system and compared its low-lying singlets with the analytically obtained eigenvalue patterns (from the effective Hamiltonian by diagonalization and by semi- 
classical calculations). Our main analytical results are summarized in Tables I and II, and our numerical results are in Tables III (for fcc) and IV (for triangular lattice). In Table IV, for the triangular lattice with $N=16$ and 28 , we have corrected some numerical mistakes in Table I of our earlier paper Ref. 1, resulting in a much better agreement between exact diagonalization and semiclassical results. As seen in Tables III and IV, our method gives good agreement between theory and numerics for cluster splittings, but with an overall shift for absolute energy values.

It seems likely that our general approach could be applied to catalog the energy and symmetry eigenvalues in exact diagonalizations of some other lattice antiferromagnets. By this approach, we mean (1) representing the low-energy classical states by several sublattice spins, with lengths proportional to the size $N$; 2 ) writing an effective Hamiltonian for the sublattice spins; and (3) analyzing this semiclassically, with especial attention to geometical phases. A caution is that the results are likely to be interesting mainly when there are multiple tunneling paths, and when the tunnel barriers are small compared to other energies (due either to classical degeneracies broken by quantum fluctuations, or else to weak anisotropies).

The motivation for a careful study of the clustering patterns of low-lying eigenstates, and thus for this paper, is that the pattern might be diagnostic of the ultimate long-range order, even in systems so small that correlation lengths and order parameters are inconclusive. Size is the key limiting factor to the usefulness of exact diagonalization for quantum many-body systems, so any approach that partially overcomes this is of interest. The eigenvalue pattern was decisive evidence of longrange order for the case of the $s=1 / 2$ nearest-neighbor triangular antiferromagnet. 39

Even richer clustering patterns occur in systems with classical ground state degeneracies and/or discrete symmetry breakings, as treated in this paper. However, studies 10 subsequent to Ref. 1 cast this partially into doubt: the eigenvalue pattern of the four-spin problem with full rotational symmetry reappeared for three spins with XY symmetry, and presumably in many systems with a discrete threefold symmetry (see Sec. V A of Ref. 10).

An alternate application of this theory would be to the burgeoning topic of small magnetic molecules, the magnetic Hamiltonian of which may often be approximated as a combination of several large subspins. 46 In the examples studied to date, the intramolecular interactions were ferromagnetic and highly anisotropic, but it is quite plausible that a molecule (or complex) with high symmetry could realize a Hamiltonian similar to one of those treated in this paper. If furthermore an experimental probe became available to measure a large number of the energy levels, this subject could be developed as chemists have developed the semiclassical treatment of molecular rotational-vibrational levels over the past twenty years.20

It would be desirable to generalize our derivation of the effective Hamiltonian to other systems with classical degeneracies, such as the fcc Type II or Type III antiferromagnets (for comparison to the exact diagonalizations of Ref. 9). This does not appear trivial. We started by finding the quantum ground-states of the infinite-range Hamiltonian $\hat{\mathcal{H}}_{\mathrm{MF}}$, and the degeneracy of its ground states was essential to our derivation, (the $p$-sublattice singlets) particularly in factoring out the energy denominators in Subsec. IV Q.

Consider, as the simplest example $\mathrm{B}$, the square lattice with sufficiently strong antiferromagnetic $J_{2}$ that the classical ordering wavevector is $(\pi, 0)$ or $(0, \pi)$. The even spins form two sublattices, oriented oppositely, so they combine to make a net singlet. Likewise the two sublattices of odd spins make a singlet, so $\hat{\mathcal{H}}_{\mathrm{MF}}$ has a unique ground state in this case. (The classical degeneracy is reflected only in the excited states of $\hat{\mathcal{H}}_{\mathrm{MF}}$.) Rather than pursue such a complicated derivation, one could instead just derive the effective Hamiltonian as in Ref. 5 (see our Eq. (4.36)), but this does not capture the system-size dependence of the coefficients.

\section{ACKNOWLEDGMENTS}

This work was supported by NSF grants DMR-9981744 (at Cornell). C.L.H. thanks G. S. Ezra and B. Bernu for useful discussions.

\section{APPENDIX A: ADDING SPINS TO GET SINGLETS}

To add up $p$ spins of length $\tilde{S}$ to get a singlet, we use the well-known decomposition of the product of two representations: $\mathcal{D}^{S_{1}} \times \mathcal{D}^{S_{2}}=\mathcal{D}^{\left|S_{1}-S_{2}\right|}+\mathcal{D}^{\left|S_{1}-S_{2}\right|+1}+\ldots+$ $\mathcal{D}^{S_{1}+S_{2}}$, where $\mathcal{D}^{S}$ is the spin $S$ irreducible representation of $S U(2)$. (Note that to get a singlet $\left(\mathcal{D}^{0}\right)$ in the preceeding expression, we must have $S_{1}=S_{2}$.) Here we show a somewhat nontrivial calculation for $p=5$ (and assume $\tilde{S}$ is an integer). One can either find the coefficent of $\mathcal{D}^{\tilde{S}}$ in $\mathcal{D}^{\tilde{S}} \times \mathcal{D}^{\tilde{S}} \times \mathcal{D}^{\tilde{S}} \times \mathcal{D}^{\tilde{S}}$, or try $\left(\mathcal{D}^{\tilde{S}} \times \mathcal{D}^{\tilde{S}} \times \mathcal{D}^{\tilde{S}}\right) \times\left(\mathcal{D}^{\tilde{S}} \times \mathcal{D}^{\tilde{S}}\right)$. Adopting the latter approach, and denoting $N_{S}$ the coefficent of $\mathcal{D}^{S}$ in $\mathcal{D}^{\tilde{S}} \times \mathcal{D}^{\tilde{S}} \times \mathcal{D}^{\tilde{S}}$, we get $N_{S}=2 S+1$ for $0 \leq S \leq \tilde{S}$ and $N_{S}=3 \tilde{S}-S+1$ for $\tilde{S}+1 \leq S \leq 3 \tilde{S}$. The number of singlets for $p=5$ is thus $\sum_{S=0}^{2 \tilde{S}} N_{S}=$ $5 \tilde{S}(\tilde{S}+1) / 2+1$. (The upper limit of the sum goes to $2 \tilde{S}$ because the maximum spin length from $\mathcal{D}^{\tilde{S}} \times \mathcal{D}^{\tilde{S}}$, the last two terms in the product of five terms, is $2 \tilde{S}$.)

\section{APPENDIX B: QUARTIC OPERATOR AND SPIN COHERENT STATE}

Here we note briefly how we compute the spin coherent state expectation value in (6.1). The straightforward way 
is to use a generating function (Eq. (6.22) in Ref. 26). We show here a second way using tensor operators (Ref. 47, Eq. (8Q)):

$$
\left\langle\theta, \phi\left|\mathcal{Y}_{L M}(\mathbf{S})\right| \theta, \phi\right\rangle=\frac{(2 S) !}{(2 S-L) ! 2^{L}} Y_{L M}(\theta, \phi) .
$$

Here $Y_{L M}(\theta, \phi)$ is the usual spherical harmonic function. $\mathcal{Y}_{L M}(\mathbf{S})$ is the spherical tensor operator obtained from the polynomial $S^{L} Y_{L M}(\theta, \phi)$ by first substituting the operators $\hat{S}_{x}, \hat{S}_{y}$, and $\hat{S}_{z}$ for classical numbers $S_{x}, S_{y}$, and $S_{z}$, and then symmetrizing the resulting operator, e.g., the polynomial $S_{x} S_{y}$ becomes the symmetrized operator $\left(\hat{S}_{x} \hat{S}_{y}+\hat{S}_{x} \hat{S}_{y}\right) / 2$.

This identity tells us that if we can write a spin operator as a linear combination of spherical tensor operators $\mathcal{Y}_{L M}(\mathbf{S})$, then its coherent state expectation value is easy to obtain. For our problem, it is easy to check the following classical identity:

$$
\begin{aligned}
& S_{x}^{4}+S_{y}^{4}+S_{z}^{4}-\frac{3}{5} S^{4}=\sqrt{\frac{4 \pi}{9}} S^{4} \\
& {\left[\frac{2}{5} Y_{4,0}(\theta, \phi)+\frac{2}{\sqrt{70}}\left(Y_{4,4}(\theta, \phi)+Y_{4,-4}(\theta, \phi)\right)\right] .}
\end{aligned}
$$

Then according to (B1), we have

$$
\begin{aligned}
& \left\langle\theta, \phi\left|\sqrt{\frac{4 \pi}{9}}\left[\frac{2}{5} \mathcal{Y}_{4,0}(\mathbf{S})+\frac{2}{\sqrt{70}}\left(\mathcal{Y}_{4,4}(\mathbf{S})+\mathcal{Y}_{4,-4}(\mathbf{S})\right)\right]\right| \theta, \phi\right\rangle \\
= & \frac{S\left(S-\frac{1}{2}\right)(S-1)\left(S-\frac{3}{2}\right)}{S^{4}}\left(S_{x}^{4}+S_{y}^{4}+S_{z}^{4}-\frac{3}{5} S^{4}\right) . \quad \text { (B3) }
\end{aligned}
$$

What is the operator on the left hand side of the previous equation? Using the table in Ref. 48, it can be checked that this spin operator is nothing but

$$
\hat{S}_{x}^{4}+\hat{S}_{y}^{4}+\hat{S}_{z}^{4}-\frac{3}{5}\left(S^{2}(S+1)^{2}-\frac{1}{3} S(S+1)\right) .
$$

On the other hand, we see that the three spherical harmonic functions involved here all have $L=4$ and according to (B1) the coefficient in front of the expectation value depends on $L$ only, i.e., the coherent state expectation values of the corresponding spherical tensors have the same coefficients. We can then start from the classical expectation value $S_{x}^{4}+S_{y}^{4}+S_{z}^{4}-\frac{3}{5} S^{4}$ and find the corresponding operator. It is straightforward to get $\hat{S}_{x}^{4}+\hat{S}_{y}^{4}+\hat{S}_{z}^{4}$, but because of operator symmetrization, $S^{4}$ goes to $S^{2}(S+1)^{2}-S(S+1) / 3$ (see Ref. 48, p.268, also Ref. 49, p.31). Putting these two terms together, we get the identity (6.1) in our paper.

${ }^{1}$ C. L. Henley and N. G. Zhang, Phys. Rev. Lett. 81, 5221 (1998).
${ }^{2}$ P. Lecheminant, B. Bernu, C. Lhuillier, and L. Pierre, Phys. Rev. B 52, 6647 (1995).

${ }^{3}$ C. L. Henley, Phys. Rev. Lett., 62, 2056 (1989).

${ }^{4}$ The theory in this paper does not apply to cases where the ground state has a macroscopic number of degrees of freedom, such as the kagomé or pyrochlore lattices with nearest-neighbor interactions.

${ }^{5}$ B. E. Larson and C. L. Henley, unpublished (1990).

${ }^{6}$ M. W. Long, J. Phys. Condens. Matt. 1, 2857 (1989).

${ }^{7}$ E. F. Shender, Sov. Phys. JETP, 56, 178 (1982).

${ }^{8}$ A. E. Jacobs and T. Nikuni, J. Phys. Condens. Matt. 10, 6405 (1998).

${ }^{9}$ K. Lefmann and C. Rischel, in press, Eur. Phys. J. B (2001).

${ }^{10}$ P. A. Houle, N. G. Zhang, and C. L. Henley, Phys. Rev. B60, 15179 (1999).

11 A. V. Chubukov and T. Jolicoeur, Phys. Rev. B46, 11137 (1992).

12 T. Oguchi, H. Nishimori, and Y. Taguchi, J. Phys. Soc. Jpn. 12, 4494 (1985).

13 C. L. Henley, J. Appl. Phys. 61, 3962 (1987)

${ }^{14} \mathrm{C}$. Lhuillier and P. Lecheminant, personal communication.

15 The Landau Fermi liquid theory, like our theory, focuses on the energy levels. The sublattice magnetization renormalization is like the wavefunction renormalization $Z$ (the overlap of the bare creation operator with the quasiparticle creation operator): $Z$ can be small compared to 1 , implying a strong renormalization of the quasiparticles, and yet the dispersion keeps the same linear dispersion as the noninteracting theory. One can relate the noninteracting theory to the Fermi liquid by an adiabatic turn-on of interactions; in our model, the turn-on of $\delta \mathcal{H}$. One expects that the eigenenergies are proportional to the corresponding eigenenergies of the mean-field (or respectively noninteracting) systems, but the coefficients in our effective Hamiltonian (respectively the Fermi liquid $m^{*}$ ) might be strongly changed.

${ }^{16}$ L. D. Landau and E. M. Lifshitz, Quantum Mechanics: Non-relativistic Theory, $§ 39$ (Pergamon, 1977).

${ }^{17}$ A familiar example of operator equivalent is related to the Lande- $g$ factor. Given an atom's magnetic moment, $\mathbf{m}=-e(\mathbf{L}+2 \mathbf{S}) / 2 \mu c$, with orbital and spin angular momenta, and form the total angular momentum, $\mathbf{J}=$ $\mathbf{L}+\mathbf{S}$, then within eigenstates of $\mathbf{J}^{2}$ and $J_{z},\left|J, M_{J}\right\rangle$, we have the following well-known relation, $\left\langle J, M_{J}^{\prime}|\mathbf{m}| J, M_{J}\right\rangle=$ $-e g\left\langle J, M_{J}^{\prime}|\mathbf{J}| J, M_{J}\right\rangle / 2 \mu c$, where $g$ is the Lande- $g$ factor. And we say that $\mathbf{m}$ and $-e g \mathbf{J} / 2 \mu c$ are operator equivalents. In the present paper, we have an operator in terms of individual spins $\mathbf{s}_{i}$, and we want to find its operator equivalent in eigenstates of sublattice spins $\mathbf{S}_{\alpha}$. It is analogous to going from $\mathbf{L}$ and $\mathbf{S}$ to $\mathbf{J}$ in the $g$-factor problem.

${ }^{18}$ E. Merzbacher, Quantum Mechanics, Chapter 16 (Wiley, New York, 1970)

19 T. Inui, Y. Tanabe, and Y. Onodera, Group Theory and Its Applications in Physics, Section 7.12 (Springer-Verlag, 1990).

${ }^{20}$ W. G. Harter and C. W. Patterson, Phys. Rev. Lett. 38, 224 (1977); J. Chem. Phys. 80, 4241 (1984).

${ }^{21}$ J. M. Robbins, S. C. Creagh, and R. G. Littlejohn, Phys. Rev. A 39, 2838 (1989). 
${ }^{22}$ V. A. Kalatsky and V. L. Pokrovsky, V.L. Europhys. Lett. 44, 539 (1998).

${ }^{23}$ D. Loss, D. P. DiVincenzo, and G. Grinstein, Phys. Rev. Lett. 69, 3232 (1992); J. von Delft and C. L. Henley, Phys. Rev. Lett. 69, 3236 (1992). See also A. Garg, Europhys. Lett. 22, 205 (1993).

${ }^{24}$ E. Lieb, Commnu. Math. Phys. 31, 327 (1973).

${ }^{25}$ R. Shankar, Phys. Rev. Lett 45, 1088 (1980).

${ }^{26}$ J. M. Radcliffe, J. Phys. A 4, 270 (1971).

27 There appears to be an alternate, one-to-one representation a single of $\left\{\hat{\mathbf{m}}_{\alpha}\right\}$ by a single sphere, as shown in Fig. 6 and footnote 26 of Ref. 10. This captures the topology of the phase space and tunneling paths, but the effective Hamiltonian on this sphere has not been related quantitatively to the one derived in the present paper.

28 There have been hints that - in single-spin problems - more accurate tunneling amplitudes would be produced with an alternative definition of the phase factor $\Phi=(S+1 / 2) \Omega$ and an alternative BS quantization condition $\Phi_{\text {orbit }}=$ $2 \pi(l+1 / 2)$, where the $S+1 / 2$ approximates $\sqrt{S(S+1)}$ and the $1 / 2$ in $l+1 / 2$ comes from the Maslov (geometric) index of the orbit, See Ref. 21, and also V. I. Belinicher, C. Providencia, and J. da Providencia, J. Phys. A 30, 5633 (1997). This was clarified very recently by M. Stone, K.-S. Park, and A. Garg, J. Math. Phys. 41, 8025 (2000).

29 The text of Ref. 1 erroneously reversed the $(1,2)$ and $(2,1)$ pattern, due to a sign error in the eigenenergy formula for the $C$ orbits on p. 5223 of that paper: it should have read $U_{C l}-2 \mid t_{C l} \cos \left[\left(2 \pi j-\operatorname{Re} \Phi_{C l}\right) / 3\right]$.

${ }^{30}$ For $N=16$ in the triangular case, the energy scale of the effective Hamiltonian is anomalously small but nonzero, both in the analytic theory and in the exact diagonalization.

${ }^{31}$ A prefactor scaling with spin length as $S^{1 / 2}$ was found earlier for tunneling between discrete energy minima. [A. Garg and G.-H. Kim, Phys. Rev. B45, 12921 (1992); J. von Delft and C. L. Henley, Phys. Rev. B 48, 965(1993).] However, since the tunneling in the present case connects two rotationally symmetric manifolds, a changed prefactor is expected [A. Auerbach and S. Kivelson, Nuc. Phys. B257[FS14], 799 (1985).]

${ }^{32}$ Ref. 1 contains (at bottom of p. 5223), a formula for the WKB exponential " $\left(8 K_{U}\right) \exp \left(-S \operatorname{Im} \Phi_{C l} / 3\right)$." This should be corrected by removing the " $S$ " factor, since that was already included in the definition of $\Phi_{C l}$.

${ }^{33}$ An alternative way to say exactly the same thing is that we have limited ourselves to total singlet states, and (independent of any semiclassical mapping) we showed in Appendix A that these are always even under permutations of the $(12)(34)$ class.

34 This argument is inspired by the notion of "induced representation" in Ref. 20, that tells us which representations of the full symmetry group contain a chosen representation of the subgroup generated by (23).

${ }^{35}$ We thank B. Bernu for unpublished data on the "axial" eigenvalues from Ref. 2. As noted in that paper, the $N=28$ triangular lattice simulation cell is incompatible with the mirror or glide symmetries. (This suggests that the 4-spin effective Hamiltonian for this lattice - if evaluated to higher order than our second-order approximation - would contain some small terms that break the odd permutation symme- tries.) Finally, the appropriate eigenvalues are unavailable for the fcc lattices because a different kind of mirror plane was used.

${ }^{36}$ P. W. Anderson, Phys. Rev. 86, 694 (1952).

${ }^{37}$ For a sufficiently large lattice antiferromagnet, the tunneling path from one discrete well to another is probably not a rigid rotation of each sublattice spin - as assumed in a $p$-spin representation - but rather is "quantum nucleation", whereby a new domain expands from a point to cover the lattice. (E. M. Chudnovsky and L. Gunther, Phys. Rev. B37, 9455 (1988).) That surely increases the magnitude of the tunnel splittings, but need not affect the geometric phase factors that govern the eigenvalue cluster splitting pattern.

${ }^{38}$ M. Gross, E. Sanchez-Velasco, and E. Siggia, Phys. Rev. 39, 2484 (1989); H. Neuberger and T. Ziman, Phys. Rev. 39, 2608 (1989).

${ }^{39}$ B. Bernu, C. Lhuillier, and L. Pierre, Phys. Rev. Lett. 69, 2590 (1992).

${ }^{40}$ A. G. Gukasov, Th. Brückel, B. Dorner, V. P. Plakhty, W. Prandl, E. F. Shender, and O. P. Smirnov, Europhys. Lett. 7, 83 (1988); T. Brückel, B. Dorner, A. G. Gukasov, V. P. Plakhty, W. Prandl, E. F. Shender, and O. P. Smirnow, Z. Phys. B 72, 477 (1988);

${ }^{41}$ Y. J. Kim, A. Aharony, R. J. Birgenau, F. C. Chou, O. Entin-Wohlman, R. W. Erwin, M. Greven, A. B. Harris, M. A. Kastner, I. Y. Korenblit, Y. S. Lee, and G. Shirane, Phys. Rev. Lett. 83, 852 (1999).

42 J. S. Smart, Effective Field Theories of Magnetism (Saunders, Philadelphia, 1966).

${ }^{43}$ C. Rischel and K. Lefmann, J. Mag. Magn. Mat. 177-181, 775 (1998).

${ }^{44}$ C. Lanczos, J. Res. Natl. Bur. Stand. Sect B 45, 255 (1950).

${ }^{45}$ J. K. Cullum and R. A. Willoughby, Lanczos Algorithms for Large Symmetric Eigenvalue Computations (Birkhäuser, Boston, 1985).

${ }^{46}$ M. I. Katsnelson, V. V. Dobrovitski, and B. N. Harmon, Phys. Rev. B 59, 6919 (1999).

${ }^{47}$ R. Gilmore, J. Phys. A 9, L65 (1976).

${ }^{48}$ M. T. Hutchings, Solid State Physics, vol. 16, F. Seitz and D. Turnbull, eds. (Academic Press, New York, 1964).

${ }^{49}$ K. Yosida, Theory of Magnetism (Spinger, Berlin, 1998). 
TABLE I. Parameters for the effective Hamiltonian

\begin{tabular}{ccc}
\hline \hline General & Triangular & Fcc \\
\hline$p$ & 4 & 4 \\
$z_{\alpha \beta}^{1}(\alpha \neq \beta)$ & 2 & 4 \\
$z_{\alpha \beta}^{2}(\alpha \neq \beta)$ & 2 & 0 \\
$z_{\alpha \alpha}^{1}$ & 0 & 0 \\
$z_{\alpha \alpha}^{2}$ & 0 & 6 \\
$\tilde{J}_{12}=\left(\sum_{m} J_{m} z_{12}^{m}\right) / \tilde{N}$ & $2\left(J_{1}+J_{2}\right) / \tilde{N}$ & $4 J_{1} / \tilde{N}$ \\
$\tilde{J}_{11}=\left(\sum_{m}^{m} J_{m} z_{11}^{m}\right) / \tilde{N}$ & 0 & $6 J_{2} / \tilde{N}$ \\
$E_{\mathrm{MF}}=(p / 2)\left(\tilde{J}_{11} \tilde{S}^{2}-\tilde{J}_{12} \tilde{S}(\tilde{S}+1)\right)$ & $-4\left(J_{1}+J_{2}\right) \tilde{S}(\tilde{S}+1) / \tilde{N}$ & $-8 J_{1} \tilde{S}(\tilde{S}+1) / \tilde{N}+12 J_{2} \tilde{S}^{2} / \tilde{N}$ \\
$K_{\alpha \beta}=\sum_{m}\left(J_{m}\right)^{2} z_{\alpha \beta}^{m}-\left(\sum_{m} J_{m} z_{\alpha \beta}^{m}\right)^{2} / \tilde{N}$ & $2\left(J_{1}^{2}+J_{2}^{2}\right)-4\left(J_{1}+J_{2}\right)^{2} / \tilde{N}$ & $4(\tilde{N}-4) J_{1}^{2} / \tilde{N}$ \\
\hline \hline
\end{tabular}

TABLE II. The series of Hamiltonians

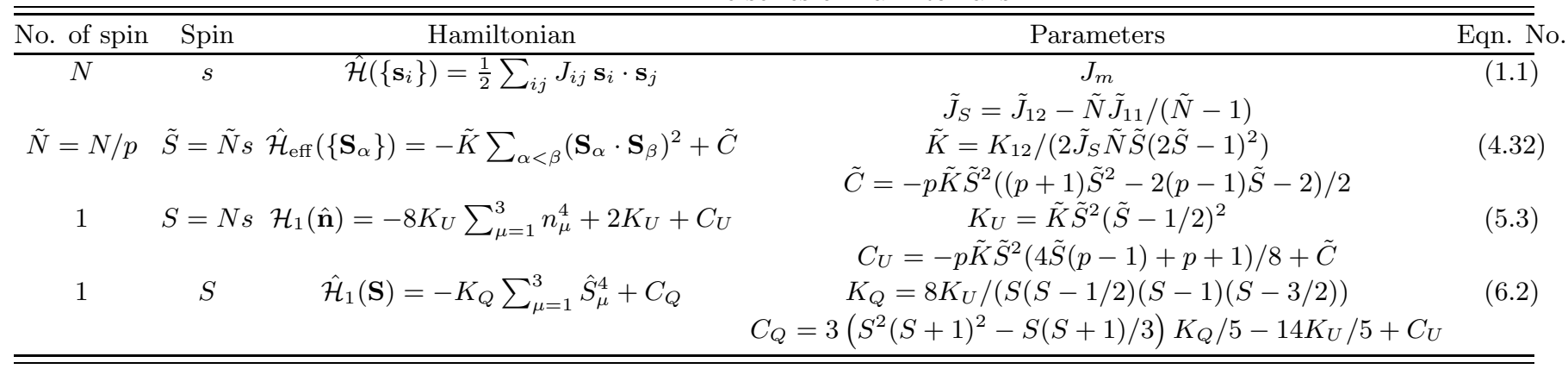

TABLE III. Eigenenergies $\Delta E=E-E_{M F}$ for $N=32$ fcc Type I $\left(J_{1}=1, J_{2}=-1\right)$. (Entries “..." denote a level where cluster labeling ambiguity occurs and are explained in Sec. Vq.)

\begin{tabular}{|c|c|c|c|c|}
\hline Orbit, degeneracies & $\begin{array}{r}\text { Exact }(1.1) \\
(\text { mean, levels) }\end{array}$ & $\begin{array}{l}4 \text {-spin } 4.32 \\
\text { (mean, levels) }\end{array}$ & $\begin{array}{l}1 \text {-spin }(6.2) \\
\text { (mean, levels) }\end{array}$ & $\begin{array}{l}\text { Semiclassic (5.3) } \\
(\text { mean })\end{array}$ \\
\hline$\overline{T_{0} 2}$ & -1.5388 & -1.0316 & -1.0659 & -1.0727 \\
\hline & $-1.7457\{-1.7126$ & $-1.2029\{-1.1492$ & $-1.1898\{-1.1436$ & -1.1718 \\
\hline$C_{1}\left\{\begin{array}{l}1 \\
1 \\
2\end{array}\right.$ & $-1.8409\left\{\begin{array}{l}{ }^{-} 1.7788 \\
-1.8720\end{array}\right.$ & $-1.2849\left\{\begin{array}{l}{ }^{-} \dot{1} .2566 \\
-1.2990\end{array}\right.$ & $-1.2519\left\{\begin{array}{l}\because \dot{1} .2361 \\
-1.2599\end{array}\right.$ & -1.2899 \\
\hline$C_{0}\left\{\begin{array}{l}2 \\
1\end{array}\right.$ & $-2.3178\left\{\begin{array}{l}-2.3151 \\
-2.3233\end{array}\right.$ & $-1.6706\left\{\begin{array}{l}-1.6703 \\
-1.6713\end{array}\right.$ & $-1.5640\left\{\begin{array}{l}-1.5639 \\
-1.5642\end{array}\right.$ & -1.5639 \\
\hline
\end{tabular}

TABLE IV. Eigenenergies $\Delta E=E-E_{M F}$ for $N=16, N=28$ triangular lattices $\left(J_{1}=1, J_{2}=0.7\right)$.

\begin{tabular}{|c|c|c|c|c|}
\hline Orbit, degeneracies & $\begin{array}{l}\text { Exact } 1.12 \\
\text { (mean, levels) }\end{array}$ & $\begin{array}{l}\text { 4-spin 4.32 } \\
\text { (mean, levels) }\end{array}$ & $\begin{array}{l}\text { 1-spin (6.2) } \\
\text { (mean, levels) }\end{array}$ & $\begin{array}{l}\text { Semiclassic (5.3) } \\
(\text { mean })\end{array}$ \\
\hline \multicolumn{5}{|l|}{$N=16$} \\
\hline$T_{0} 2$ & -0.0756 & -0.0730 & -0.0792 & -0.0824 \\
\hline$C_{0}\left\{\begin{array}{l}2 \\
1\end{array}\right.$ & $-0.1359\left\{\begin{array}{l}-0.1331 \\
-0.1417\end{array}\right.$ & $-0.1366\left\{\begin{array}{l}-0.1343 \\
-0.1412\end{array}\right.$ & $-0.1178\left\{\begin{array}{l}-0.1170 \\
-0.1193\end{array}\right.$ & -0.1176 \\
\hline \multicolumn{5}{|l|}{$N=28$} \\
\hline$T_{0} 2$ & -1.8769 & -1.9191 & -1.9929 & -2.0135 \\
\hline$C_{1}\left\{\begin{array}{l}2 \\
1\end{array}\right.$ & $-2.0106\left\{\begin{array}{l}-2.0022 \\
-2.0275\end{array}\right.$ & $-2.3361\left\{\begin{array}{l}-2.2993 \\
-2.4097\end{array}\right.$ & $-2.2821\left\{\begin{array}{l}-2.2601 \\
-2.3261\end{array}\right.$ & -2.3699 \\
\hline$C_{0}\left\{\begin{array}{l}1 \\
2\end{array}\right.$ & $-2.3843\left\{\begin{array}{l}-\overline{2} .3840 \\
-2.3845\end{array}\right.$ & $-3.1576\left\{\begin{array}{l}-\overline{3} .1539 \\
-3.1595\end{array}\right.$ & $-2.9257\left\{\begin{array}{l}-2.9246 \\
-2.9262\end{array}\right.$ & -2.9252 \\
\hline
\end{tabular}

